ACCEPTED ON APJ.

Preprint typeset using $\mathrm{LT}_{\mathrm{E}} \mathrm{X}$ style emulateapj v. 6/22/04

\title{
QUASAR LUMINOSITY FUNCTIONS FROM JOINT EVOLUTION OF BLACK HOLES AND HOST GALAXIES
}

\author{
A. Lapi ${ }^{1}$, F. Shankar ${ }^{3}$, J. MaO ${ }^{1}$, G.L. Granato ${ }^{2,1}$, L. Silva ${ }^{4}$, G. De Zotti $^{2,1}$, L. Danese ${ }^{1,2}$ \\ Draft version June 22, 2018
}

\begin{abstract}
We show that our previously proposed anti-hierarchical baryon collapse scenario for the joint evolution of black holes and host galaxies predicts quasar luminosity functions at redshifts $1.5 \lesssim z \lesssim 6$ and local properties in nice agreement with observations. In our model the quasar activity marks and originates the transition between an earlier phase of violent and heavily dust-enshrouded starburst activity promoting rapid black hole growth, and a later phase of almost passive evolution; the former is traced by the submillimeter-selected sources, while the latter accounts for the high number density of massive galaxies at substantial redshifts $z \gtrsim 1.5$, the population of Extremely Red Objects, and the properties of local ellipticals.
\end{abstract}

Subject headings: galaxies: formation - galaxies: evolution - quasars: general

\section{INTRODUCTION}

Observations in the optical and in the X-ray bands have shown since a couple of decades that very bright quasars (QSOs) occur very soon in the history of the Universe, and that their average luminosity significantly declines from redshift $z \sim 2$ toward the present (e.g., Mathez 1978; Giacconi 1985; Schmidt \& Green 1986). Modern observations have confirmed and detailed such a picture (e.g., Fan et al. 2004, 2006; Richards et al. 2005, 2006; Cristiani et al. 2004; Barger et al. 2005; Tozzi et al. 2001; Brandt $\&$ Hasinger 2005), measuring QSO luminosity functions (LFs) up to $z \sim 6$.

Supermassive black holes (BHs) were found to be ubiquitous in the centers of spheroidal galaxies (Kormendy \& Richstone 1995). Moreover, a narrow relationship between the central supermassive BH mass and the stellar mass/luminosity of the spheroidal/bulge component has been firmly established by observations (e.g., Magorrian et al. 1998; Marconi \& Hunt 2003). The BH mass also strictly correlates with the velocity dispersion of the spheroidal component (e.g., Ferrarese \& Merritt 2000; Gebhardt et al. 2000; Tremaine et al. 2002). Contrariwise, it has been shown that the BH mass is on average at least $10^{2}$ times smaller in late-type irregular and/or spiral galaxies than in ellipticals with the same luminosity (see Salucci et al. 2000). All together these observations witness the strict connection between the mass of the BH and that of the old stars formed at $z \gtrsim 1$ within the host galaxy. Recently, the studies of QSO host galaxies have been extended to high redshift (e.g., Dunlop et al. 2003; Floyd et al. 2004), with the general result that they are typically early-types.

The stellar populations of ellipticals are old and have similar ages/formation histories, although there is some trend for more massive systems to be on average older and more metal-rich (Sandage \& Visvanathan 1978; Bernardi et al. 1998; Trager et al. 2000; Terlevich \& Forbes 2002; Thomas et al. 2005). A color-magnitude relation is well established, indicating that more luminous spheroids are redder (Bower et al. 1992). The widely accepted interpretation is that brighter objects are richer in metals and that the spread of their star formation epochs is small enough to avoid smearing of their colors. In fact, the slope of the relation seems not to change with redshift (Ellis et al. 1997; Kodama et al. 1998), supporting this view. Note, however, that the trend of having on average older stellar populations in more massive ellipticals may also constitute an important ingredient (e.g., Jørgensen et al. 1999; Caldwell, Rose \& Concannon 2003; Nelan et al. 2005).

The star formation history of spheroidal galaxies is mirrored in the Fundamental Plane (e.g., Jedrzejewski et al. 1987; Dressler et al. 1987) and in its evolution with redshift. Ellipticals adhere to this plane with a surprisingly low orthogonal scatter (around 15\%), as expected for a homogeneous family. Furthermore, recent studies (e.g., Treu et al. 2002; van der Wel et al. 2004; Holden et al. 2005) suggest that ellipticals, both in the field and in the clusters, lay on the fundamental plane up to $z \approx 1$, consistent with massive spheroids being old and quiescent. Progenitors of local massive early-type galaxies have been identified through K-band and Spitzer surveys at substantial redshifts, $z \gtrsim 1$. Direct evidence that massive galaxies with stellar content $M_{\star} \gtrsim 10^{11} M_{\odot}$ were in place at $z \gtrsim 2$ is provided by recent K-band surveys (e.g., Cimatti et al. 2002; Kashikawa et al. 2003; Fontana et al. 2004; Bundy, Ellis \& Conselice 2005). The space density of Extremely Red Objects (EROs) at $z \gtrsim 3$ is only a factor about $5-10$ less than that at $z \sim 1$ (e.g., Tecza et al. 2004). This implies a phase of extremely high star formation rate (SFR), from several hundreds to thousands solar masses per year, taking place at high $z$. Such a phase is also witnessed by the sub-mm galaxy counts (e.g., Chapman et al. 2003; 2005).

On the theoretical side, the hierarchical clustering paradigm led to the development of various semi-analytic models for galaxy formation. These generally share the basic assumption that gravity is the main driver in shaping the structure and morphology of galaxies. In this scenario, the gas cools and form stars following the collapse of dark matter (DM) halos. In the standard cosmology, small DM objects form first and merge together to make larger ones. This scenario then implies that large ellipticals form late, by mergers of disk/bulge systems made primarily of stars, recently indicated as 'dry mergers' (e.g., Naab et al. 2006).

${ }^{1}$ Astrophysics Sector, SISSA/ISAS, Via Beirut 2-4, I-34014 Trieste, Italy.

2 INAF, Osservatorio Astronomico di Padova, Vicolo dell' Osservatorio 5, I-35122 Padova, Italy.

${ }^{3}$ Dept. of Astronomy, Ohio State University, $140 \mathrm{~W} 18^{\text {th }}$ Avenue, OH 43210-1173 Columbus, USA.

${ }^{4}$ INAF, Osservatorio Astronomico di Trieste, Via Tiepolo 11, I-34131 Trieste, Italy. 
But the early appearance of QSOs with high luminosity and presumably huge BH mass is at variance with expectations from this framework (e.g., Bromley et al. 2004). This apparent contradiction may be solved by invoking a higher efficiency in forming massive BHs in smaller galaxy halos at higher redshift (e.g., Haehnelt \& Rees 1993; Haiman \& Loeb 1998; Wyithe \& Loeb 2003; Mahmood et al. 2005).

Granato et al. (2001, 2004), instead, explored the possibility of reconciling the observed 'downsizing' of QSOs and spheroidal galaxies with the bottom-up DM hierarchy (see also Somerville et al. 2004; Baugh et al. 2005; Croton et al. 2006; Hopkins et al. 2006). This has been done by developing a simple model that incorporates the main physical aspects of the DM and baryons residing within galactic halos. This new approach emphasized the role of energy feedback processes both from supernovae (SNae), capable of unbinding the gas in low-mass systems, and from the QSO phase of the supermassive BHs, capable of ejecting gas from the largest objects (see Di Matteo, Springel \& Hernquist 2005; Lapi, Cavaliere \& Menci 2005). These feedback processes can actually reverse the formation sequence of visible galaxies with respect to that of DM halos. Hence large galaxies end their star formation, and have their BHs shining as QSOs early on. On the contrary, the star formation and the QSO phase are more prolonged in smaller halos (hence the name of 'Anti-hierarchical Baryon Collapse', or ABC, scenario).

While in previous works we focused on reproducing the properties of spheroidal galaxies, in this paper we explore the constraints on model parameters set by the redshift-dependent LFs of QSOs. Our plan is as follows: in $\S 2$ we briefly recall the main features of the model by Granato et al. (2004); in $\$ 3$ we describe the procedure adopted to compute the QSO LFs, the supermassive BH mass function, and other galactic observables; $\S 4$ is devoted to the illustration of our results; in $\S 5$ we make a critical comparison of our findings with other models in the literature; finally, in $\S 6$ we summarize and discuss our conclusions.

Throughout this work we adopt the cosmology indicated by the WMAP data (Bennett et al. 2003; Spergel et al. 2006), i.e., a flat Universe with matter density $\Omega_{M} \approx 0.27$, baryon density $\Omega_{b} \approx 0.044$ and Hubble constant $H_{0} \approx 71 \mathrm{~km} \mathrm{~s}^{-1} \mathrm{Mpc}^{-1}$.

\section{THE MODEL FROM THE GROUND UP}

This paper is based on the semi-analytic model developed by Granato et al. (2004), which follows the evolution of baryons within proto-spheroids through simple and physically grounded recipes.

We defer the interested reader to that paper for a full account of the physical justification and a detailed description of the model. Here we provide a short summary of its main features, focusing on the aspects relevant to our discussion on QSO LFs and $\mathrm{BH}$ demographics.

\subsection{The dark matter sector}

As for the treatment of the DM in galaxies, the model follows the standard hierarchical clustering framework, taking also into account the results by Wechsler et al. (2002), and Zhao et al. (2003a; 2003b). Their simulations have shown that the growth of a halo occurs in two different phases: a first regime of fast accretion in which the potential well is built up by the sudden mergers of many clumps with comparable masses; and a second regime of slow accretion in which mass is added in the outskirts of the halo, without affecting the central region where the galactic structure resides.

This means that the halos harboring a massive elliptical galaxy once created, even at high redshift, are rarely destroyed. At low redshifts they are incorporated within groups and clusters of galaxies. Support to this view comes from studies of the mass structure of elliptical galaxies, which are found not to show strong signs of evolution since redshift $z \approx 1$ (Koopmans et al. 2006). Note that, as pointed out in $\S 1$, the BH mass is strictly correlated with properties (mass and velocity dispersion) of the old stars in massive early-type galaxies, formed at least $8 \mathrm{Gyr}$ ago (see Thomas et al. 2005) and, as a consequence, in massive galaxy halos virialized at $z \gtrsim 1.5$.

We deal just with the latter redshift range $(z \gtrsim 1.5)$, where a good approximation of the halo formation rates is provided by the positive term in the cosmic time derivative of the cosmological mass function (e.g., Haehnelt \& Rees 1993; Sasaki 1994). For DM halos with mass $M_{\mathrm{vir}}$ at time $t_{\mathrm{vir}}$, these formation rates are given by

$$
\frac{\mathrm{d}^{2} N_{\mathrm{ST}}}{\mathrm{d} t_{\mathrm{vir}} \mathrm{d} M_{\mathrm{vir}}}=\left[\frac{a \delta_{c}\left(t_{\mathrm{vir}}\right)}{\sigma^{2}\left(M_{\mathrm{vir}}\right)}+\frac{2 p}{\delta_{c}\left(t_{\mathrm{vir}}\right)} \frac{\sigma^{2 p}\left(M_{\mathrm{vir}}\right)}{\sigma^{2 p}\left(M_{\mathrm{vir}}\right)+a^{p} \delta_{c}^{2 p}\left(t_{\mathrm{vir}}\right)}\right]\left|\frac{\mathrm{d} \delta_{c}}{\mathrm{~d} t_{\mathrm{vir}}}\right| N_{\mathrm{ST}}\left(M_{\mathrm{vir}}, t_{\mathrm{vir}}\right),
$$

where $N_{S T}\left(M_{\mathrm{vir}}, t\right)$ is the Sheth \& Tormen $(1999,2002)$ version of the PS mass function (Press \& Schecter 1974). In the above equation, $a=0.707$ and $p=0.3$ are constants obtained from comparison of the mass function with the outcome of $N$-body simulations; $\sigma\left(M_{\mathrm{vir}}\right)$ is the mass variance of the primordial perturbation field, computed from the Bardeen et al. (1986) power spectrum with correction for baryons (Sugiyama 1995), and normalized to $\sigma_{8} \approx 0.84$ on a scale of $8 h^{-1}$ Mpc; $\delta_{c}\left(t_{\mathrm{vir}}\right)$ is the critical threshold for collapse, extrapolated from the linear perturbation theory. Note that our adoption of the Sheth $\&$ Tormen mass function to construct the rates is mandatory, since it is well-known that the canonical Press \& Schechter theory strongly underpredicts the number of massive halos, particularly at the high redshifts relevant for the computation of the QSO LFs.

We confine our analysis to galaxy halo masses $M_{\text {vir }}^{\text {min }} \gtrsim 2 \times 10^{11} M_{\odot}$, since we are interested in following the evolutionary history of bright QSOs and of their host galaxies. At the other end, weak lensing observations (e.g., Kochanek \& White 2001; Kleinheinrich et al. 2004) and kinematical measurements (e.g., Kronawitter et al. 2000; Gerhard et al. 2001) suggest an upper limit in galaxy halo mass $M_{\mathrm{vir}}^{\max } \approx 2 \times 10^{13} M_{\odot}$. The same limit is also indicated by the theoretical analysis of Cirasuolo et al. (2005) on the velocity dispersion function of early-type galaxies. These limits in mass and redshift ensure that the positive cosmic time derivative of the halo mass function is a good approximation to the formation rate of DM halos, as the negative term is negligible.

Note, however, that since we are interested in DM halos associated to a single galaxy, we need to correct the Sheth \& Tormen halo mass function by: (i) accounting for the possibility that a DM halo contains various sub-halos each hosting a galaxy; (ii) removing halos corresponding to galaxy systems rather than to individual galaxies. We deal with (ii) by simply cutting-off the 

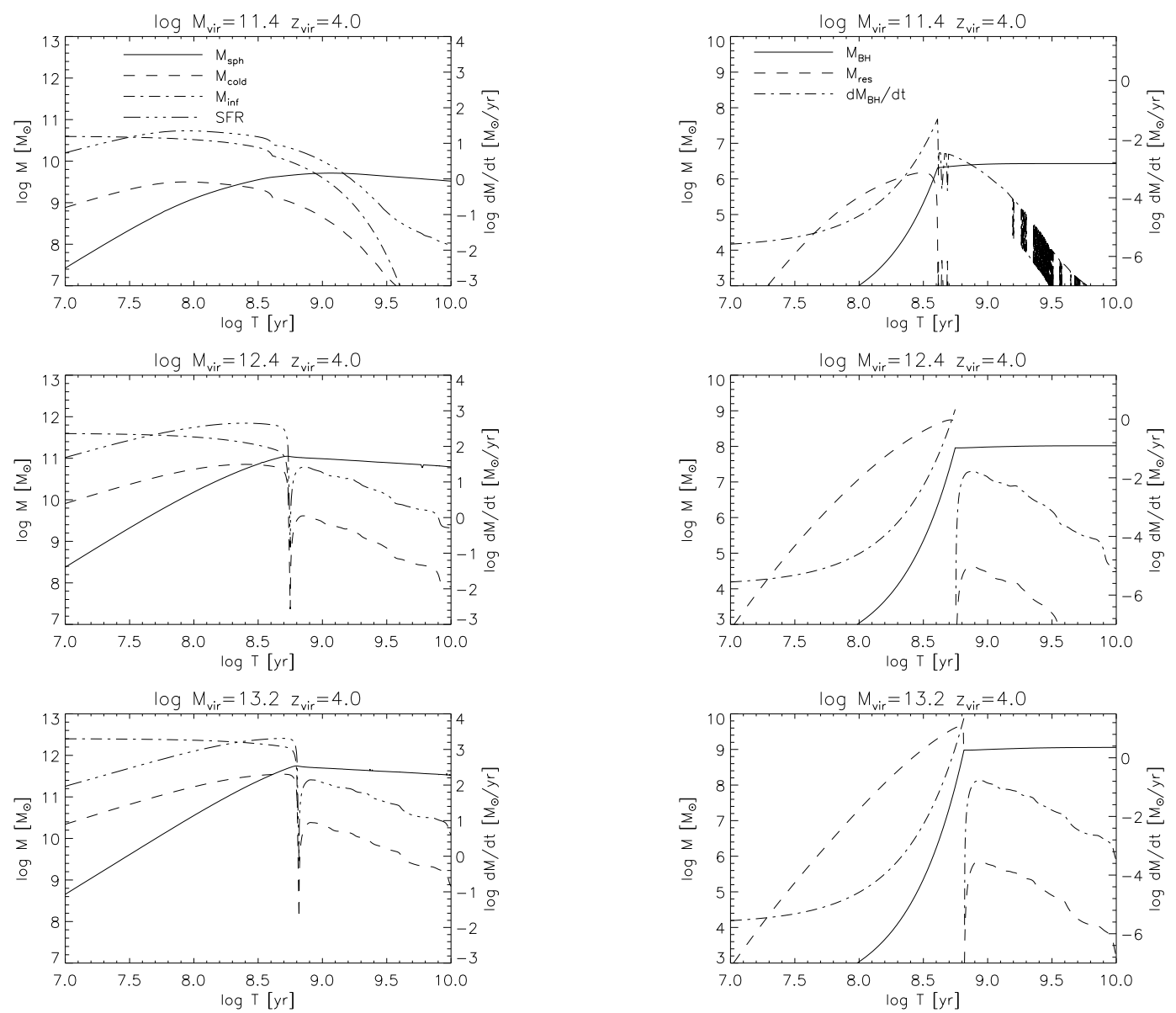

FIG. 1.- Left panel: dependence on galactic age of the stellar mass, infalling mass, cold gas mass, and star formation rate within halos of various masses, virialized at redshift 4. Right panel: same for the $\mathrm{BH}$ mass, reservoir mass, and $\mathrm{BH}$ accretion rates.

halo mass function at $M_{\mathrm{vir}}^{\max } \approx 2 \times 10^{13} M_{\odot}$, beyond which the probability of having multiple galaxies within a halo quickly becomes very high (e.g., Magliocchetti \& Porciani 2003). As for (i), we add the sub-halo mass function, following the procedure described Vale \& Ostriker (2004; 2006) and Shankar et al. (2006), and using the fit to the sub-halo mass function at various redshifts provided by van den Bosch, Tormen \& Giocoli (2005). However we have checked that for the masses and redshifts relevant here $\left(z \sim 1.5-6\right.$ and $\left.M_{\text {vir }} \sim 2 \times 10^{11} M_{\odot}-2 \times 10^{13} M_{\odot}\right)$, the total mass function (sub-halo + halo) differs from the halo mass function by less than $5 \%$. Thus, using simply the halo mass function to derive the DM halo formation rates is a good approximation.

\subsection{The baryonic sector}

The physics governing the evolution of the baryons is much more complex than for the DM. The main features of our model can be summarized as follows (see Granato et al. 2004 and Cirasuolo et al. 2005 for additional details).

During or soon after the formation of the host DM halo, the baryons falling into the newly created potential well are shockheated to the virial temperature. The hot gas is (moderately) clumpy and cools quickly especially in the denser central regions, triggering a strong burst of star formation. The radiation drag due to starlight acts on the gas clouds, reducing their angular momentum. As a consequence, a fraction of the cool gas can fall into a reservoir around the central supermassive $\mathrm{BH}$, and eventually accretes onto it by viscous dissipation, powering the nuclear activity. The energy fed back to the gas by SN explosions and $\mathrm{BH}$ activity regulates the ongoing star formation and the $\mathrm{BH}$ growth. Eventually, most of the gas is unbound from the DM potential well, so that the star formation and the $\mathrm{BH}$ activity come to an end on a timescale shorter for the more massive galaxies.

In Appendix A and Table A1 we present the basic equations and parameters controlling the evolution of the baryonic component in our model, once the halo mass and the virialization redshift are given. These equations can be numerically integrated to yield, among others, the SFR and the accretion rate onto the central BH as function of cosmic time. In Fig. 11we plot the basic outputs of the model.

Initially, the cooling is rapid and the star formation is very high; thus the radiation drag is efficient in accumulating mass into the reservoir. The BH starts growing from an initial seed with mass $10^{2} M_{\odot}$ already in place at the galactic center. Since there is plenty of material in this phase, the accretion is Eddington (or moderately super-Eddington) limited (e.g., Small \& Blandford 1992; Blandford 2004). This regime goes on until the energy feedback from the BH is strong enough to unbind the gas from the potential well, a condition occurring around the peak of the accretion curve. Subsequently, the SFR drops substantially, the 

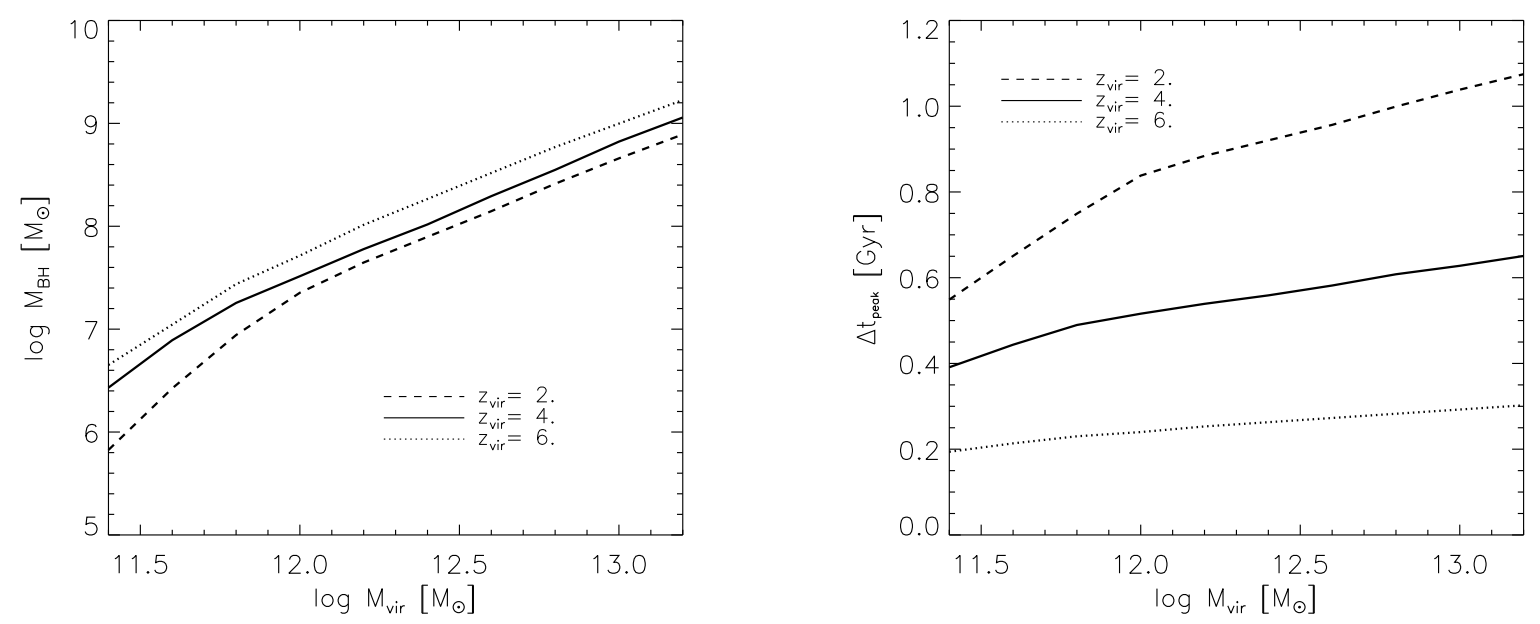

FIG. 2.- Left panel: BH mass at the accretion peak as a function of the host galaxy virial mass. Right panel: time lag between the host galaxy virialization and the peak in the $\mathrm{BH}$ accretion rate, as a function of the host virial mass.

radiation drag becomes inefficient, the storage of matter in the reservoir and the accretion onto the $\mathrm{BH}$ decrease by a large factor. The drop is very pronounced for massive halos $M_{\mathrm{vir}} \gtrsim 10^{12} M_{\odot}$, while for smaller masses a smoother declining phase can continue for several Gyrs, and the BH and stellar masses can further increase by a substantial factor.

Before the peak, radiation is highly obscured by the surrounding dust. In fact, these proto-galaxies are extremely faint in the UV-optical rest frame and are more easily selected at (sub-)mm wavelengths. The nuclear emission is heavily obscured too; however, since the absorption significantly decreases with increasing X-ray energy of photons, it is easier to detect it in hard $\mathrm{X}$-ray bands. On the other hand, in the proximity of the accretion peak, i.e., when the central supermassive BH is massive and powerful enough to remove most of the gas and dust from the surroundings, the active nucleus shines as an optical QSO.

The list of the observations well fitted by the model is presented in Table A2, along with a brief description of the main underlying assumptions. In order to translate the SFR and the mass in stars into more directly observable quantities such as broad-band luminosities, including dust extinction, we exploit the GRASIL code (Silva et al. 1998, see http://web.pd.astro.it/granato). As for the QSOs, the model yields bolometric luminosities that are translated into luminosities in a given band by means of appropriate bolometric corrections. The epoch-dependent luminosity functions are derived combining the results obtained for halos of fixed mass and virialization redshift with the halo formation rates presented in $\S 2.1$.

\section{THE MODEL AT WORK}

As a first step we want to properly translate the DM formation rates given by Eq. (1) into BH formation rates. This calls for a relation between the halo mass $M_{\mathrm{vir}}$ and the BH mass $M_{\bullet}$. In Fig. 2(left panel) we plot the one obtained by solving the equations presented in Appendix A, that can be well approximated by

$$
M_{\bullet} \approx 8 \times 10^{6} \frac{\left(M_{\mathrm{vir}} / 2.2 \times 10^{11} M_{\odot}\right)^{3.97}}{1+\left(M_{\mathrm{vir}} / 2.2 \times 10^{11} M_{\odot}\right)^{2.7}}\left(\frac{1+z_{\mathrm{vir}}}{7}\right) M_{\odot} .
$$

This relationship is very close to that found, at $z=0$, by Shankar et al. (2006) by comparing the local BH mass function with the galaxy halo mass function. However, this is only an average relationship and we do expect a significant scatter around it, since the values of the parameters in the equations of Appendix A may naturally vary from halo to halo. In the following we assume that the above relation $\log M_{\bullet}-\log M_{\text {vir }}$ holds on average with a gaussian dispersion $\Delta \log M_{\bullet}$. Therefore we convert the halo formation rates into $\mathrm{BH}$ formation rates through the convolution

$$
\frac{\mathrm{d}^{2} N_{\mathrm{BH}}}{\mathrm{d} t_{\mathrm{vir}} \mathrm{d} M_{\bullet}}=\left.\int \mathrm{d} \log M_{\bullet}^{\prime}\left|\frac{\mathrm{d} M_{\mathrm{vir}}}{\mathrm{d} M_{\bullet}}\right|_{M_{\bullet}^{\prime}} \frac{\mathrm{d}^{2} N_{\mathrm{ST}}}{\mathrm{d} t_{\mathrm{vir}} \mathrm{d} M_{\mathrm{vir}}}\right|_{M_{\mathrm{vir}}\left(M_{\bullet}^{\prime}\right)} \frac{e^{-\left(\log M_{\bullet}^{\prime}-\log M_{\bullet}\right)^{2} / 2\left(\Delta \log M_{\bullet}\right)^{2}}}{\sqrt{2 \pi\left(\Delta \log M_{\bullet}\right)^{2}}} .
$$

It will be shown below that the dispersion is an important ingredient when comparing model predictions to observations.

Note that despite the redshift dependence of the $M_{\bullet} / M_{\text {vir }}$ ratio (see Eq. [2]), our model predicts a relatively weak evolution of the BH-bulge mass ratio. This is because, as mentioned in $\$ 2.2$, the growth of the BH mass is controlled by the SFR through the radiation drag and the SN feedback; in turn, the feedback from the QSO can eventually sweep out the gas halting both the star formation and the accretion on the $\mathrm{BH}$, especially in massive galaxies. In the latter structures the stellar and $\mathrm{BH}$ masses grow (and stop growing) in parallel, while in smaller objects where only SN feedback is effective the BH-bulge mass ratio turns out to depend slightly on $z$ (see Fig. 5 of Cirasuolo et al. 2005).

\subsection{QSO luminosity functions and BH mass function}


The QSO LFs can now be computed. Up to its peak, the BH bolometric light curve can be well approximated by the simple exponential form

$$
L(t)=\frac{\lambda M_{\bullet} c^{2}}{t_{\text {Edd }}} e^{\left(t-t_{\mathrm{vir}}-\Delta t_{\text {peak }}\right) / \tau_{\mathrm{ef}}} \theta_{H}\left(t_{\mathrm{vir}}+\Delta t_{\text {peak }}-\Delta t_{\mathrm{vis}} \lesssim t \lesssim t_{\mathrm{vir}}+\Delta t_{\text {peak }}\right) .
$$

Here $t_{\text {Edd }} \approx 4 \times 10^{8} \mathrm{yr}$ is the Eddington timescale, and $\tau_{\mathrm{ef}} \approx \eta t_{\mathrm{Edd}} /(1-\eta) \lambda$ is the $e$-folding time in terms of the BH mass-energy conversion efficiency $\eta$ and of the Eddington ratio $\lambda$. The Heaviside function ${ }^{5} \theta_{H}$ specifies that the QSO shines unobscured only during the time interval $\Delta t_{\text {vis }}$ before the peak of its light curve. This means that, as discussed in $\S 2.2$, at the time $t_{\text {vir }}+\Delta t_{\text {peak }}-\Delta t_{\text {vis }}$ the $\mathrm{BH}$ is massive enough for its energy feedback to remove most of surrounding gas and dust, letting the active nucleus to be visible. In this work we have used an Eddington ratio $\lambda$ slightly rising toward high $z$, and specifically $\lambda=4$ for $z \gtrsim 6, \lambda=3$ for $5 \lesssim z \lesssim 6, \lambda=1.7$ for $3 \lesssim z \lesssim 5, \lambda=1$ for $2 \lesssim z \lesssim 3$, and $\lambda=0.8$ for $1.5 \lesssim z \lesssim 2$. The empirical formula $\lambda(z) \approx-1.15+0.75(1+z)$ works as well in the redshift range $1.5 \lesssim z \lesssim 6$ (see also Appendix A and Table A1). This assumption is discussed in $\S 6$.

There are three timescales in Eq. (4): the virial time $t_{\text {vir }}$ depends on cosmology; the peak time $\Delta t_{\text {peak }}$ is obtained by solving the system of equations reported in Appendix $\mathrm{A}$; the visibility time $\Delta t_{\text {vis }}$, dependent on dust and gas absorption, is taken as a parameter, since its computation is challenging for semi-analytical models.

The QSO LF at a time $t$ and luminosity $L$ is computed by summing up the contributions of all the sources which virialize at epochs $t_{\mathrm{vir}} \lesssim t$ and shine at the time $t$ with luminosity $L$. One has

$$
\Phi(L, t)=\int_{t-\Delta t_{\text {peak }}}^{t-\Delta t_{\text {peak }}+\Delta t_{\text {vis }}} \mathrm{d} t_{\mathrm{vir}} \int \mathrm{d} M_{\bullet} \frac{\mathrm{d}^{2} N_{\mathrm{BH}}}{\mathrm{d} t_{\mathrm{vir}} \mathrm{d} M_{\bullet}} \delta_{D}\left(L-\frac{\lambda M_{\bullet} c^{2}}{t_{\text {Edd }}} e^{\left(t-t_{\mathrm{vir}}-\Delta t_{\text {peak }}\right) / \tau_{\mathrm{ef}}}\right),
$$

where $\delta_{D}$ indicates the Dirac delta function. The time delay $\Delta t_{\text {peak }}$ provided by our code ranges from $0.2 \mathrm{Gyr}$ at redshift $z \gtrsim 5$ where $\lambda \approx 4$ to 1 Gyr at redshifts $z \lesssim 2$, where $\lambda \lesssim 1$ (see Fig. 2 right panel).

In our model, the declining phase of the QSO light curve can be neglected for halo masses above few $\times 10^{12} M_{\odot}$, because for them the QSO feedback is so powerful to remove suddenly most of the accreting gas from the surroundings. As a consequence, after the peak the accretion rate drops abruptly to values much lower (by factors of $10^{-2}$ at least; see Fig. 1) than the Eddington rate, and the radiation process becomes much less efficient (for a review see Blandford 2004). We have checked that such radiatively inefficient stages provide a negligible contribution to the QSO LFs, in accord with the findings by Yu \& Lu (2004) based on the comparison between the observed QSO LFs and the local supermassive BH mass function.

On the contrary, for halo masses smaller than $10^{12} M_{\odot}$ and especially at redshifts $z \lesssim 2$, the $\mathrm{BH}$ accretion rate decays more gently after the peak, and up to few Gyrs are required before it lowers to levels of $\sim 10^{-2}$ the Eddington rate, becoming radiatively inefficient. This declining phase is consistent with observations of moderate QSO activity in relatively typical early-type galaxies, with old stellar populations (e.g., Kauffmann et al. 2003). This regime, which adds to the visibility time defined above (that refers to the rising phase of the bolometric light curve), is included in our computation of the QSO LFs. We find that it contributes significantly to the faint end of the X-ray QSO LFs (see also Hopkins et al. 2006b) and to the X-ray number counts (see Granato et al. 2006 for details). On the other hand, the optical luminosities are generally below those corresponding to the standard definition of optical QSOs $\left(M_{B} \leq-23\right)$; therefore the declining phase has a lower effect on optical LFs.

The last step of our computation is the conversion of the bolometric QSO LFs computed above to the optical and hard X-ray bands through the appropriate bolometric corrections (e.g., Elvis et al. 1994; Ueda et al. 2003; Vignali, Brandt \& Schneider 2003; McLure \& Dunlop 2004; Barger et al. 2005; Richards et al. 2005, 2006). For the optical band we use $L / L_{B}=f_{B}$ with $f_{B} \approx 10$. For the hard X-ray band $(2-10 \mathrm{keV})$ we take into account the observed luminosity dependence of $L / L_{X}=f_{X}$, given by $f_{X} \approx k_{X}^{1 /\left(\beta_{X}+1\right)}\left(L / 10^{43} \mathrm{erg} \mathrm{s}^{-1}\right)^{\beta_{X} /\left(\beta_{X}+1\right)}$, with $k_{X} \approx 17$ and $\beta_{X} \approx 0.43$ (see also Shankar et al. 2004).

The epoch-dependent $\mathrm{BH}$ mass function is given by

$$
\Psi\left(\log M_{\bullet}, t\right)=\int_{0}^{t} \mathrm{~d} t_{\mathrm{vir}} \frac{\mathrm{d}^{2} N_{\mathrm{BH}}}{\mathrm{d} t_{\mathrm{vir}} \mathrm{d} \log M_{\bullet}} .
$$

\subsection{Related galactic properties}

Our model aims at predicting, at the same time, both the QSO LFs at various redshifts, and the properties of host galaxies.

Using the DM halo formation rates of Eq. (1) we can compute the galaxy LF in a given band as

$$
\Phi\left(\log L_{\mathrm{i}}, t\right)=\int_{0}^{t} \mathrm{~d} t_{\mathrm{vir}} \frac{\mathrm{d}^{2} N_{\mathrm{ST}}}{\mathrm{d} t_{\mathrm{vir}} \mathrm{d} M_{\mathrm{vir}}}\left|\frac{\mathrm{d} M_{\mathrm{vir}}}{\mathrm{d} \log L_{\mathrm{i}}}\right|,
$$

where $L_{\mathrm{i}}$ is the luminosity in the $\mathrm{i}$-th band. Similarly, the velocity distribution function is given by

$$
\Omega(\log \sigma, t)=\int_{0}^{t} \mathrm{~d} t_{\text {vir }} \frac{\mathrm{d}^{2} N_{\mathrm{ST}}}{\mathrm{d} t_{\text {vir }} \mathrm{d} M_{\text {vir }}}\left|\frac{\mathrm{d} M_{\text {vir }}}{\mathrm{d} \log \sigma}\right| .
$$

To derive the Jacobians we use: (i) the GRASIL code, which evaluates the luminosity of a galaxy as a function of time once the dust content and the SFR (obtained by solving the system of equations in Appendix A) is specified; (ii) the scaling law for

\footnotetext{
${ }^{5}$ The Heaviside function $\theta_{H}$ is defined by
}

$$
\theta_{H}(x)= \begin{cases}1, & \text { if } x \text { is true } \\ 0, & \text { otherwise }\end{cases}
$$



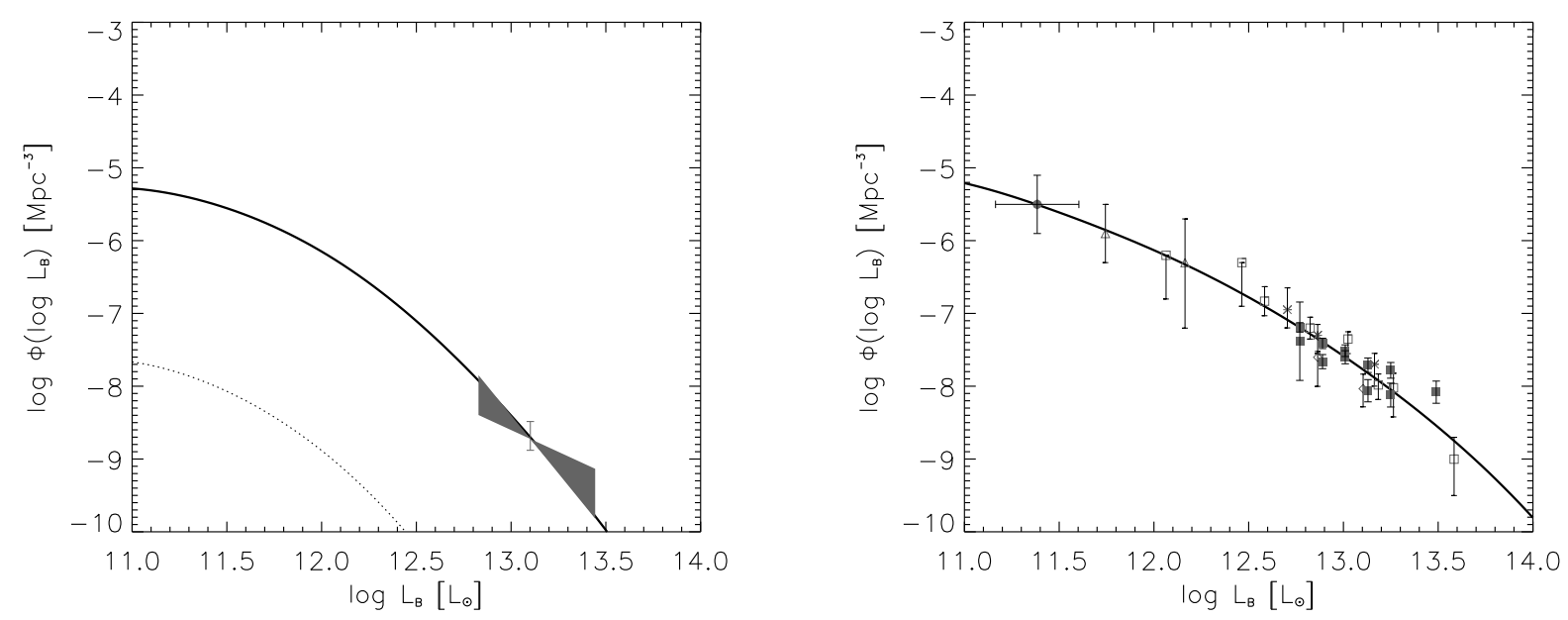

FIG. 3.- Left panel: predicted QSO LF in the optical B-band at $z=6$ (solid line) and $z=8$ (dotted line) for an Eddington ratio $\lambda=4$, and a visibility time $\Delta t_{\mathrm{vis}} \approx 5 \times 10^{7} \mathrm{yr}$. Data points are from Fan et al. (2004). Right panel: QSO LF in the optical B-band at $z=5$ for $\lambda=3$ and $\Delta t_{\mathrm{vis}} \approx 5 \times 10^{7}$ yr. Data points are from Kennefick et al. (1995, open squares); Fan et al. (2001, diamonds and asterisks); Wolf et al. (2003, triangles); Cristiani et al. (2004, circles); Richards et al. (2006, filled squares).

the virial velocity $V_{\mathrm{vir}} \equiv\left(G M_{\mathrm{vir}} / R_{\mathrm{vir}}\right)^{1 / 2} \propto M_{\mathrm{vir}}^{1 / 3}\left(1+z_{\mathrm{vir}}\right)^{1 / 2}$; (iii) the ratio between virial velocity and stellar velocity dispersion, $\sigma=0.55 V_{\text {vir }}$, found by Cirasuolo et al. (2005). The combination of these three ingredients yields a magnitude-velocity dispersion (Faber-Jackson) relation in good agreement with that $\left(M_{r^{*}}=-7.5 \log \sigma-5\right)$ observationally derived by Sheth et al. (2003) through the bisector method (see also Cirasuolo et al. 2005).

Exploiting the $\Phi\left(L_{\mathrm{i}}, t\right)$, it is straightforward to compute the galaxy counts and the related redshift distributions. These estimates have been worked out in particular for sub-mm and K-band selected galaxies, whose counts and redshift distributions proved to be particularly challenging for competing semi-analytic models. We defer the interested reader to the papers by Granato et al. (2004) and Silva et al. (2005) for the description of the computational strategy, based on the GRASIL code, and of the results.

\section{RESULTS}

\subsection{QSO Luminosity Functions}

An important feature of the model is the prediction of a pre-QSO phase lasting $\Delta t_{\text {peak }} \sim 15-20 e$-folding times (for the most massive objects), during which the mass of the central BH is increasing exponentially. Meanwhile the host galaxy is forming stars at extremely high rates (cf. Appendix A and Fig. 11 see also Sanders et al. 1988; Croom et al. 2006; Hopkins et al. 2006) and is therefore very bright at far-IR and (sub-)mm wavelengths.

In Figs. 3 6 we show that our model provides a very good fit to the optical and hard X-ray QSO LFs over the full redshift range over which it applies $(z \gtrsim 1.5)$. Due to the delay $\Delta t_{\text {peak }}$ between the halo virialization epoch and the peak of the BH accretion, the QSOs shining at the cosmic time $t_{H}(z)$ are associated to halos virializing at an earlier time $t_{\text {vir }} \approx t_{H}(z)-\Delta t_{\text {peak }}<t_{H}(z)$.

This is an important point since, at high redshifts and for massive halos, $\Delta t_{\text {peak }}$ corresponds to a substantial fraction of the cosmic time (see Fig. 2 right panel), and the DM halo formation rates at $t_{\text {vir }}$ are significantly lower than at $t_{H}(z)$. This is illustrated by Fig. 3 (left panel), where we also show the predicted optical LF of QSOs at $z=8$. The precipitous drop of the number density of bright QSOs, compared to $z=6$, is due to the fact that, for $t_{H}(z=8), \Delta t_{\text {peak }} \approx 0.2$ Gyr corresponds to $\Delta z \approx 2$, so that the abundance of bright QSOs reflects that of virialized massive halos at $z \approx 10$, which is far lower than at $z \approx 8$.

The flattening of the LFs at the low end is due to the flatter slope of the formation rate of less massive halos (see Eq. [1]) and to the weak dependence of the peak time, $\Delta t_{\text {peak }}$, on the halo mass (cf. Fig. 2).

The highest luminosity portion of the LFs corresponds to the high tail of the distribution of $M_{\bullet} \operatorname{around}$ the mean $\log M_{\bullet}-\log M_{\mathrm{vir}}$ relation, at large halo masses. The fit to the data is obtained with a Gaussian distribution with $\operatorname{dispersion} \Delta \log M_{\bullet} \approx 0.3$. As can be seen in Fig. 4 (right panel), for $\Delta \log M_{\bullet} \approx 0$ the cutoff in galaxy halo mass (cf. $\S 2.1$ ) would yield a drastic drop around $L_{B} \approx 10^{13.2} L_{\odot}$. We have checked that, for a given mass and virialization redshift of the host halo, a reasonable variation of the physical parameters from structure to structure can account for such a scatter. For example, at $z \approx 6$ and $M_{\text {vir }} \approx 2 \times 10^{13} M_{\odot}$ it can be achieved if the clumping factor $\mathcal{C}$ varies by a factor of 2 , or the seed BH mass varies by a factor of 10 , or the strength of the QSO feedback $\epsilon_{Q S O}$ varies by a factor of a few. Mahmood et al. (2005) also noted this problem, and empirically solved it by inserting a Lorentzian tail in the BH formation rate for halo masses above $10^{13.2} M_{\odot}$.

The observations by Richards et al. (2006, see their Fig. 20) show that the number density of very luminous QSOs with $M_{1450} \lesssim-27$ peaks between $z=2$ and $z=3$. Our model reproduces this trend. Specifically, the rise from high redshift to $z \approx 2.5$ is due to the strong increase of the formation rate of very massive halos, which overwhelms the effect of the decrease of the $\mathrm{BH}$ mass associated to a given $M_{\text {vir }}$ (see Eq. [2]). But at redshifts $z \lesssim 2$ the latter effect dominates, causing the fall in the number density of bright sources.

To reproduce the optical data, we adopt a visibility time $\Delta t_{\text {vis }} \approx 5 \times 10^{7}-10^{8} \mathrm{yr}$, the longer value being favored at redshifts $z \lesssim 2$. The X-ray LFs require visibility times around $\Delta t_{\mathrm{vis}} \approx 3 \times 10^{8}$ yr at $1.5 \lesssim z \lesssim 3$. The visibility time turns out to be a 

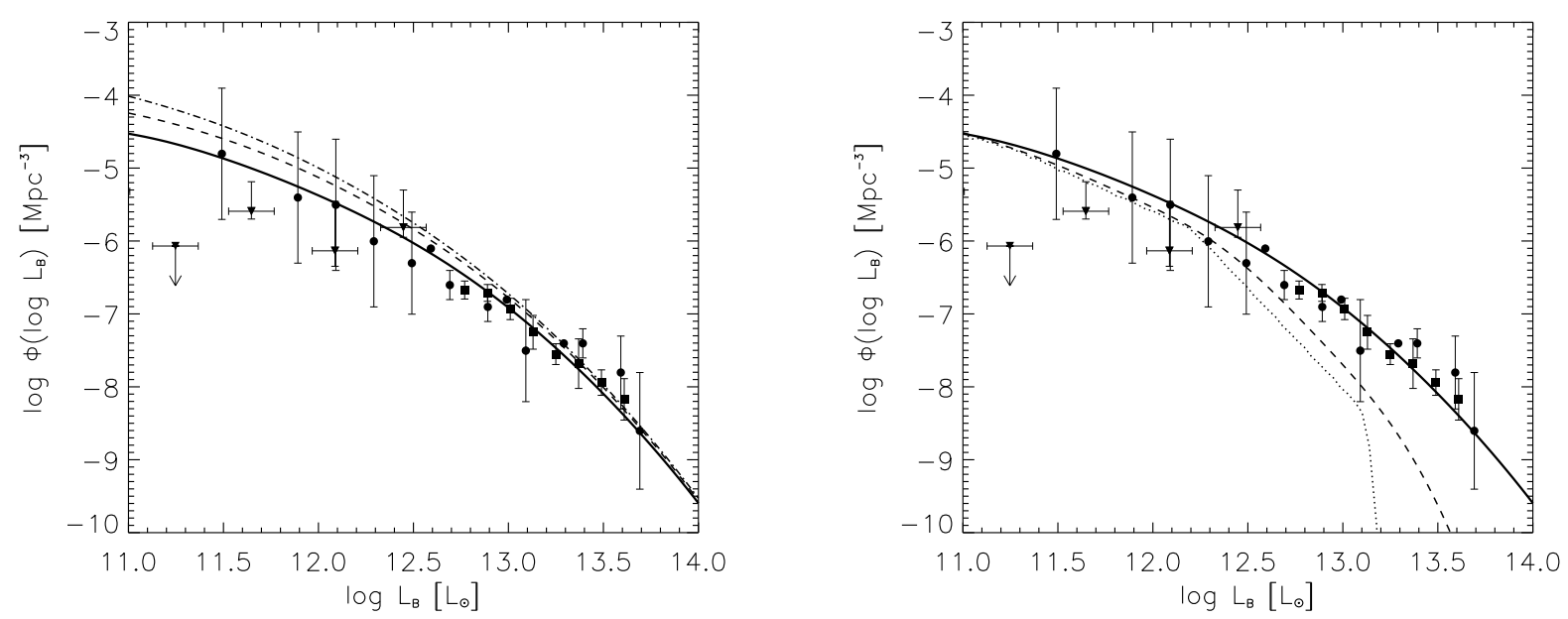

FIG. 4.— Left panel: predicted QSO LF in the optical B-band at redshift 3 for $\lambda=1.7$ and different values of the visibility time: $\Delta t_{\text {vis }} \approx 5 \times 10^{7}$ yr (best fit, solid line), $\Delta t_{\mathrm{vis}} \approx 10^{8} \mathrm{yr}$ (dashed) and $\Delta t_{\mathrm{vis}} \approx 3 \times 10^{8} \mathrm{yr}$ (dot-dashed). Right panel: effect of the scatter around the mean $\log M_{\bullet}-\log M_{\mathrm{vir}}$ relation. The solid curve is the same as in the left panel and corresponds to a scatter of $0.3 \mathrm{dex}$, as assumed throughout this paper. The dashed curve is for a scatter of 0.15 dex and the dotted one for zero scatter. In both panels data are from Pei (1995, circles), Hunt et al. (2004, triangles), and Richards et al. (2006, squares).

factor of 5-10 shorter than the time $\Delta t_{\text {peak }}$ spent by the $\mathrm{BH}$ to grow to its final mass. This implies that absorption has a key role in determining the duration of the visible QSO phase; in fact, large amounts of dust are present in the pre-QSO phase, as witnessed by sub-mm observations. Note that such a phase can also be probed through hard X-ray observations, than can detect the emission from the growing supermassive BH. Actually such emission has already been revealed from the center of sub-mm selected galaxies (Alexander et al. 2005; Borys et al. 2005); a specific discussion of this issue is presented by Granato et al. (2006).

As stated in $\S 3.1$, the accretion rate onto the central BH has been taken to be at most slightly super-Eddington, $\lambda=\dot{M}_{\bullet} / \dot{M}_{\bullet}^{\text {Edd }} \lesssim$ 4, as expected when it is limited by radiation pressure (see Small \& Blandford 1992; King \& Pounds 2003). Observational evaluations of the Eddington ratio $\lambda$ for high luminosity QSOs with $L \gtrsim 10^{47} \mathrm{erg} \mathrm{s}^{-1}$ at redshifts $z \gtrsim 2$ have been obtained by Warner, Hamann, \& Dietrich (2004) and Vestergaard (2004). These authors find a significant fraction of QSOs radiating at mildly super-Eddington powers. On the other hand, at lower redshifts and luminosities the emission seems to be limited to $\lambda \lesssim 1$ (McLure \& Dunlop 2004; Kollmeier et al. 2006). Note that these results are to be taken cautiously. In fact, they are based on the observed FWHM of the $\mathrm{H}_{\beta}$, MgII and CIV broad lines and on an empirical relation linking the size of the broad-line region to the QSO luminosity. On top of that, a normalization to the $M_{\bullet}-\sigma$ relation is needed to set the scaling factor in the equation yielding the BH mass. This coefficient depends crucially on the kinematic and geometrical properties of the broad line region itself (see Onken et al. 2004). Thus the inferred Eddington ratios suffer from large observational and systematic uncertainties, which amount to a factor of up to 10 on individual objects, and up to 4 on statistical samples (see Vestergaard \& Peterson 2006). In our model a variation of the Eddington ratio $\lambda$ with the redshift is necessary to fit the QSOs LFs (see $\S 6$ for a discussion of this point).

If radiation pressure keeps the BH growth at around the Eddington limit, outflows with mass rates $\dot{M}_{w} \sim \dot{M}_{\text {Edd }}$ are expected, as shown in Appendix A. In massive galaxies the outflows remove most of the cold and infalling gas. The model predicts the existence of clouds of chemically enriched gas flowing out from the host galaxies (the expelled cold gas). Such outflows have indeed been detected by spectroscopic studies of narrow absorption lines associated to QSOs (Srianand \& Petitjean 2000; D' Odorico et al. 2004). The average metallicity of the gas expelled from large galaxies $\left(M_{\mathrm{vir}} \gtrsim 10^{12} M_{\odot}\right)$ is $Z \sim 1-2 Z_{\odot}$, a value that after proper dilution contributes to the metal abundance $Z \sim Z_{\odot} / 3$ of the intergalactic medium in the central regions of clusters (see the review by Voit 2004). Note, however, that gas clouds coming from the innermost regions can have strongly supersolar metallicities.

The accreted mass function predicted by our model is plotted in Fig. 7 and is found to be in good agreement with the empirical determinations by Shankar et al. (2004) and Marconi et al. (2004). This shows that about $60 \%$ of the relic supermassive BH mass density is in place already at $z \gtrsim 1.5$. The rest of the mass is accreted at later times onto BHs with final mass $M_{\bullet} \lesssim 10^{8} M_{\odot}$. This result agrees with observations, showing that at $0.5 \lesssim z \lesssim 1.5$ QSOs of relatively low luminosity, $L_{X} \lesssim 3 \times 10^{44} \mathrm{erg} \mathrm{s}^{-1}$, provide about $60 \%$ of the X-ray background (Ueda et al. 2003). The $40 \%$ in mass accreted at $z \lesssim 1.5$ can account for this fraction if the ratio of X-ray to bolometric luminosity increases with decreasing redshift and/or luminosity, as suggested by observations (e.g., Wilkes 1994; Vignali et al. 2003; Strateva et al. 2005). The accreted mass function is consistent with the Soltan (1982) argument. We have checked this by redoing the calculations of Shankar et al. (2004) with the Eddington ratios adopted in this paper, and exploiting the Ueda et al. (2003) LFs corrected for very obscured sources.

\subsection{Host galaxy properties}

The model, interfaced to the GRASIL code, provides also good fits to a variety of galactic properties. Most of the results have been presented in previous papers (Granato et al. 2004, Cirasuolo et al. 2005, Silva et al. 2005) and are summarized in Table A2. 

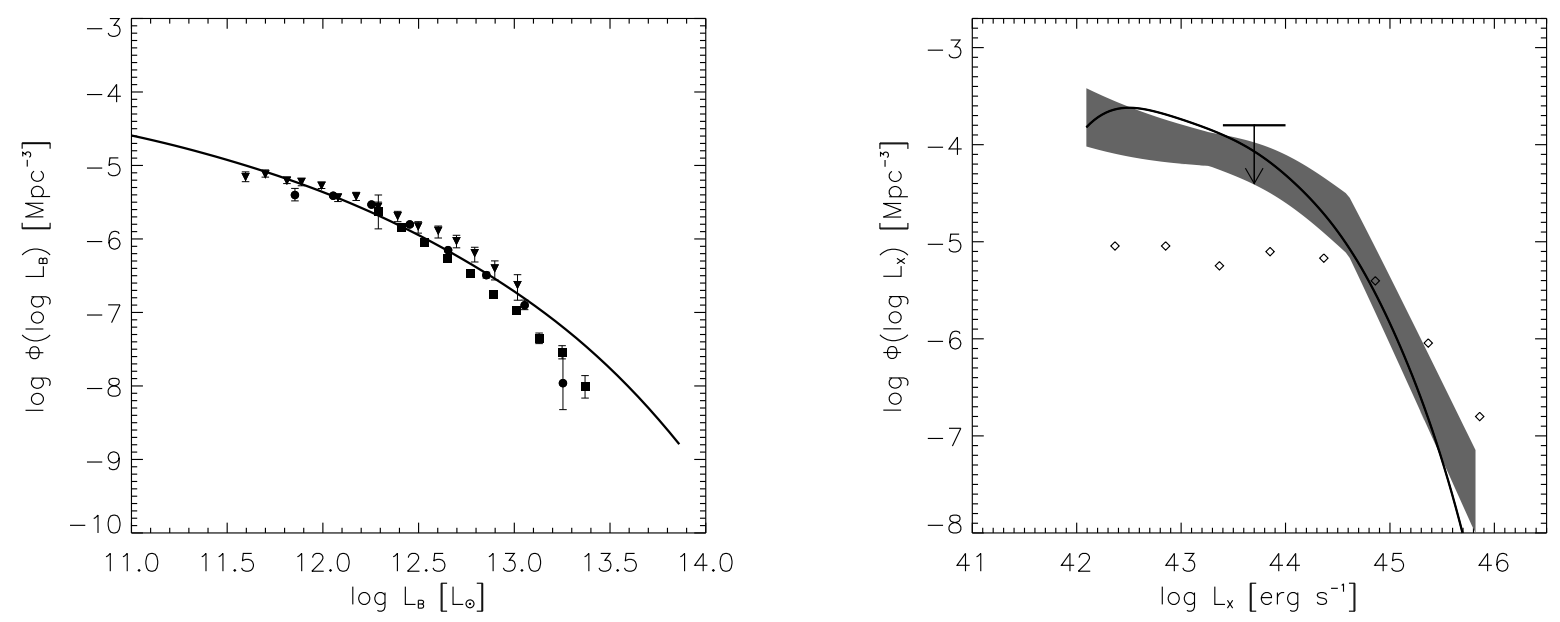

FIG. 5. - Left panel: The QSO LF in the optical B-band at redshift 2 where $\lambda=1$, with the visibility time set to $\Delta t_{\text {vis }} \approx 10^{8}$ yr; data points are from Croom et al. (2004; circles), from Richards et al. (2005; triangles), and from Richards et al. (2006; squares). Right panel: The QSO LF in the hard X-ray band (2-10 keVs) at redshift 2 where $\lambda=1$, with the visibility time set to $\Delta t_{\mathrm{vis}} \approx 3 \times 10^{8} \mathrm{yr}$; data are from Ueda et al. (2003, shaded area), from Barger et al. (2005, diamonds), and from La Franca et al. (2005, arrow).

Note that the current set of parameters differs from the one adopted in Granato et al. (2004), but the general scenario and in particular the results concerning the galaxy properties are essentially unaffected.

Figure 1 shows that small and large halos exhibit quite different behaviors. In halos with masses $M_{\mathrm{vir}} \lesssim 10^{12} M_{\odot}$ the interplay between SFR, BH accretion, and respective feedback processes, limits the growth of the central $\mathrm{BH}$, which never reaches the power needed to remove all the gas. Thus the SFR is prolonged over times $\Delta t_{\star} \gtrsim \Delta t_{\text {peak }}$, i.e., a substantial amount of stars is formed after the peak of the QSO activity. Contrariwise, in large galaxies with $M_{\mathrm{vir}} \gtrsim 10^{12} M_{\odot}$ the growth of the $\mathrm{BH}$ produces winds, which are able to stop star formation in a short time $\Delta t_{\star} \approx \Delta t_{\text {peak }}$, see Fig. 22 (right panel).

On the other hand, in the pre-QSO phase the SFR varies from several hundred to several thousand solar masses per year, within halos of mass ranging from $10^{12}$ to $10^{13} M_{\odot}$. Almost all the associated power is emitted in the far-IR. The model fits the $850 \mu \mathrm{m}$ counts; in particular, it predicts a large surface density $N(\gtrsim 1 \mathrm{mJy}, z \gtrsim 5) \approx 600$ (sq. deg. $)^{-1}$ of pre-QSO host galaxies at substantial redshift among bright objects selected at $850 \mu \mathrm{m}$ (Silva et al. 2005). This large density (compared to that of the QSOs at the same redshift) is mainly ascribed to the fact that the pre-QSO phase lasts a factor of 10 longer than the QSO phase, i.e., $\Delta t_{\text {peak }} / \Delta t_{\text {vis }} \approx 10$ at high redshifts.

A direct consequence of the extremely high SFR in massive halos is the early appearance of galaxies with large mass in stars ( $M_{\star} \propto \mathrm{SFR} \times \Delta t_{\text {peak }}$ at high redshifts). These galaxies, at redshifts corresponding to cosmological times $t_{H} \gtrsim t_{\text {vir }}+\Delta t_{\text {peak }}$, have already gone through the QSO phase and are evolving passively, i.e., without major additions of newly formed stars or large mass accretions onto the central $\mathrm{BH}$.

Note that the $850 \mu \mathrm{m}$ counts can be reproduced also adopting SFRs much lower than predicted by our model, provided that a flatter initial mass function is assumed (Baugh et al. 2005). The latter approach yields a much lower number density of massive, high-redshift galaxies. For example, the predicted number density of galaxies with $M_{\star} \gtrsim 10^{11} M_{\odot}$ becomes negligibly small at redshift $z \gtrsim 2.5$, while in our model it decreases by only a factor of 2 compared to that at $z \approx 1.5$, and it is still significant at $z \approx 5$. Stronger constraints on this number density would therefore help in discriminating between the two scenarios (e.g., Tecza et al. 2004; Fontana et al. 2004; Greve et al. 2005; Caputi et al. 2006; Bouwens \& Illingworth 2006).

Our model also naturally accounts for the observed trend of more massive spheroids being richer in metals (see Thomas et al. 2005). In our model the behavior of the average stellar metallicity $\left\langle Z_{*}\right\rangle$ as a function of the host halo mass is well described by the approximate expression $\left\langle Z_{*}\right\rangle \approx-0.247+0.023 \log \left(M_{\mathrm{vir}} / M_{\odot}\right)$. This dependence can be understood as follows. In massive galaxies the SN feedback can remove only a small fraction of the cold gas involved in star formation, which is rapidly metalenriched due to the high SFR. When the QSO reaches the peak of its activity, the energy released removes from the halo most of both the enriched cold gas and of the infalling metal-poor gas (see also Cox et al. 2006). Afterwards, the gas infall and the star formation cease, and the metallicity of the gas does not change anymore. In small galaxies SN explosions are more efficient at removing the enriched cold gas but not the infalling metal-poor gas. Thus the former can continuously be replaced by the latter, and the metallicity of the cold gas remains low. As a result large galaxies exhibit stellar populations with larger metal content than smaller galaxies do. We also stress that the short $\Delta t_{\star} \approx 0.2-0.5 \mathrm{Gyr}$ for $z_{\mathrm{vir}} \geq 4$ is responsible for the observed enhancement of $\alpha$ elements in massive galaxies (see also Romano et al. 2002; Granato et al. 2004), since the Fe enrichment is strictly related to the explosions of Type-I $a$ SNae and these are delayed by about 1 Gyr.

The power of the QSO outflows ensures that only about $30 \%$ of the initial cosmic baryons are turned into stars in large halos $\left(M_{\mathrm{vir}} \gtrsim 10^{12} M_{\odot}\right)$. For smaller halos the long lasting action of SNae is able to remove an even larger fraction of gas, letting only less than $10 \%$ of the initial gas to turn into stars (Granato et al. 2004).

Cirasuolo et al. (2005) found that the observed velocity dispersion function of spheroidal galaxies (Sheth et al. 2003) and the Faber-Jackson relation can be reproduced under the hypotheses that the old stellar populations are located in galaxy halos 

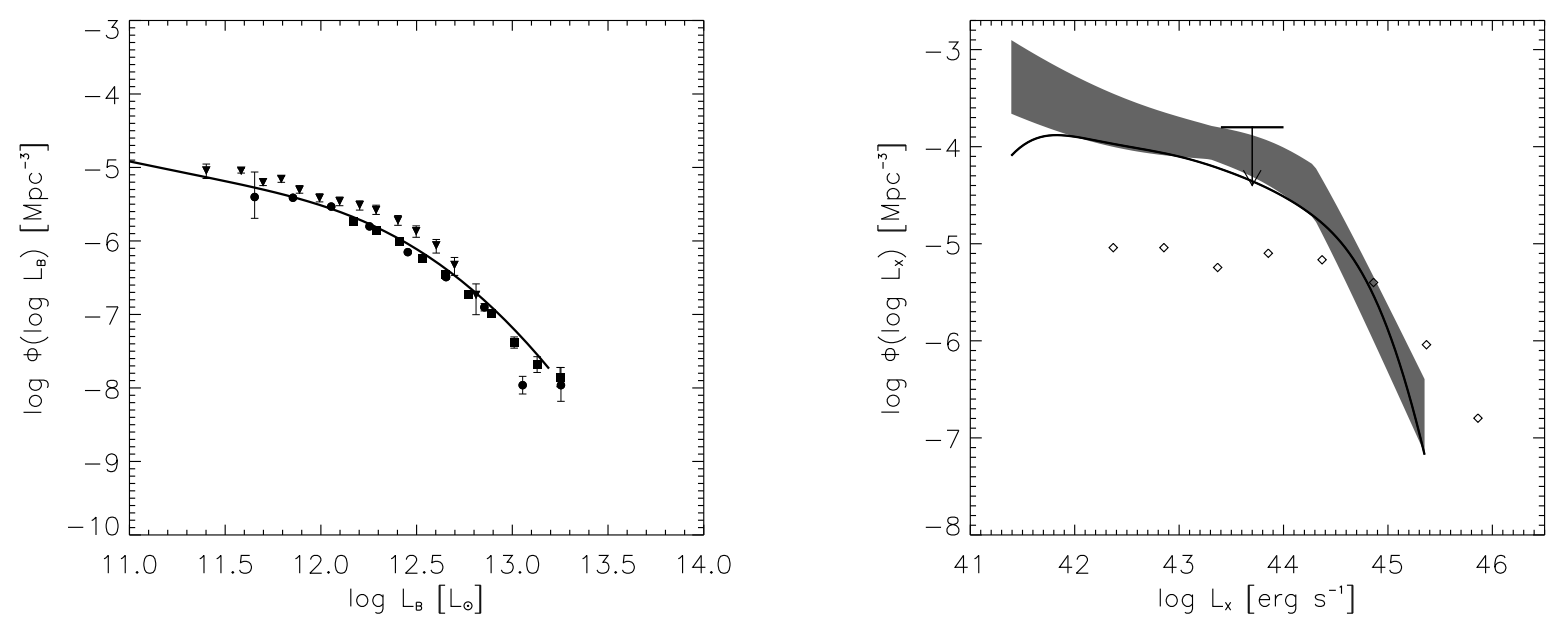

FIG. 6. - Left panel: QSO LF in the optical B-band at $z=1.5$, for $\lambda=0.8$ and $\Delta t_{\mathrm{vis}} \approx 10^{8}$ yr. The data are from Croom et al. (2004; circles), Richards et al. (2005; triangles), and Richards et al. (2006; squares). Right panel: QSO LF in the hard X-ray band $(2-10 \mathrm{keV})$ at $z=1.5$ for $\lambda=0.8$ and $\Delta t_{\text {vis }} \approx 3 \times 10^{8}$ yr. The data are from Ueda et al. (2003, shaded area), Barger et al. (2005, diamonds), and La Franca et al. (2005, arrow).

virialized at redshift $z \gtrsim 1.5$ and that the relation $\sigma \approx 0.55 V_{\text {vir }}$ holds; here the velocity dispersion $\sigma$ refers to the old stellar populations in the central galaxy regions. They also showed (cf. their Fig. 4) that the same $\sigma-V_{\text {vir }}$ relation combined with the $M_{\bullet}-M_{\text {vir }}$ relation for redshift $z \gtrsim 1.5$, fits the data in the $M_{\bullet}-\sigma$ plane, with their dispersion (Ferrarese \& Merritt 2000; Gebhardt et al. 2000; Tremaine et al. 2002).

Our model predicts a steepening of the relationship in this plane at low masses, since for small halos SNae damp down the SFR, thus hampering the growth of the reservoir around the seed BHs and eventually of the BHs themselves (cf. Eq. AA4; see also Granato et al. 2004). This steepening is fully consistent with the upper limits on the BH mass for M33 (Gebhardt et al. 2001) and NGC205 (Valluri et al. 2005) obtained by direct dynamical measurements, although the indirect determinations by Greene \& Ho (2006) suggest a constant slope and a wide scatter at small BH masses. On the other hand, we should also keep in mind that the low $\mathrm{BH}$ mass portion of the diagram might just reflect the distribution of $\mathrm{BH}$ seeds possibly created by merging of smaller $\mathrm{BHs}$ during the fast accretion epoch and be only weakly affected by the mass accretion. In addition, low BH masses may be easily increased by substantial factors during later accretion phases, when host galaxies are disk-(rather than bulge-)dominated.

\section{COMPARISON WITH PREVIOUS MODELS}

Accounting for the wealth of data on the QSO and host galaxy LFs and on their statistical properties and relationships is a severe challenge for analytical, semi-analytical and numerical galaxy formation and evolution models.

The standard methodology adopted by analytical models to estimate the QSO LFs is to convert the DM halo formation rates into $\mathrm{BH}$ formation rates through a relationship between the mass of the supermassive $\mathrm{BH}$ and that of the host halo. Then a description of the QSO light curve (usually assuming Eddington-limited accretion) is adopted to derive the QSO luminosity. The QSO LFs are then built up according to the approximate expression

$$
\Phi(L, z) \approx \Delta t_{\text {vis }} \frac{\mathrm{d}^{2} N_{\mathrm{ST}}}{\mathrm{d} t_{\text {vir }} \mathrm{d} M_{\text {vir }}}\left|\frac{\mathrm{d} M_{\text {vir }}}{\mathrm{d} M_{\bullet}}\right|\left|\frac{\mathrm{d} M_{\bullet}}{\mathrm{d} L}\right| .
$$

The above equation corresponds to Eq. (5), under the assumptions that the duty-cycle of QSO activity $\Delta t_{\text {vis }}$ is much shorter than the cosmological time and that the QSO appears as soon as the host halo virializes, i.e. $\Delta t_{\text {peak }} \approx 0$.

Wyithe \& Loeb (2003) and Mahmood et al. (2004) estimated the QSO LFs as a function of cosmic time by assuming: (i) no delay between virialization and QSO peak luminosity; (ii) a visibility time $t_{\mathrm{vis}} \approx t_{\text {disc }}$ where the dynamical time of the galactic disc is $t_{\text {disc }}=0.035 R_{\text {vir }} / V_{\text {vir }} \approx 7 \times 10^{7}(1+z)^{-3 / 2} \mathrm{yr}$; (iii) halo formation rates derived from the extended Press \& Schechter theory (Lacey \& Cole 1993); (iv) the $M_{\bullet}-M_{\text {vir }}$ relation deduced from the relations $M_{\bullet}-\sigma$ and $\sigma-M_{\text {vir }}$ observed by Ferrarese (2002).

The first and second assumptions are quite critical at high redshift. If the time scale of QSO activity is associated to the dynamical timescale of a galactic disc, one has first to wait for the disc to be built up, which implies a delay time $\Delta t \gtrsim \max \left[t_{\text {cool }}, t_{\mathrm{dyn}}\right]$, where both times are much larger than $t_{\text {disc }}$.

The specification of the DM halo formation rates is also a crucial step for analytical models. Most of the papers in the literature adopt the extended Press \& Schechter theory (Bond et al. 1991). In this paper we have used instead the rates provided by the positive part of the time derivative of the Sheth \& Tormen mass function. Note that in both cases the rates are given by the product of the mass function (Press \& Schechter or Sheth \& Tormen, respectively) by a factor, weakly dependent on time (Kitayama \& Suto 1996). As a matter of fact, mass functions derived from numerical simulations are much better approximated by the Sheth \& Tormen than by the Press \& Schechter theory; the latter at high redshifts and at the large halo masses, relevant for QSOs, can be smaller by a factor of 10 (e.g., Springel et al. 2005).

As for the last assumptions recalled above, Wyithe \& Loeb (2003) and Mahmood et al. (2004) assume a $M_{\bullet}-M_{\text {vir }}$ relationship steeper and more rapidly evolving than ours. The overall result is that, on average, they predict much more massive BHs at high 


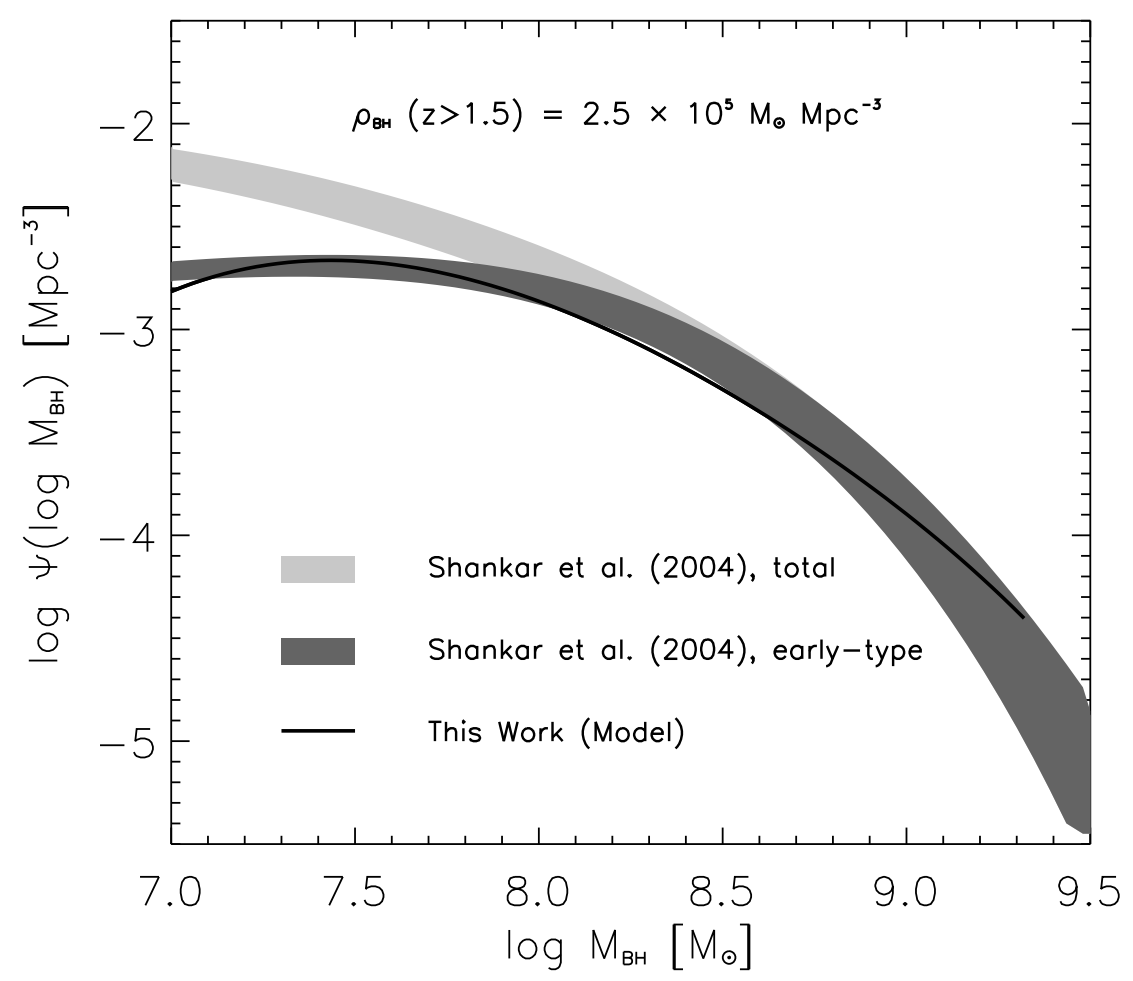

FIG. 7.-Comparison of the predicted mass function of supermassive BHs associated with massive spheroidal galaxies (solid line) with observational estimates (Shankar et al. 2004). The dark shaded area refers to the local supermassive BH mass function considering only early-type galaxies; the light shaded area includes also bulges of late-types.

redshift and for large halo masses (see also the discussion in Shankar et al. 2006). For example, at $z=6$ and for $M_{\text {vir }}=10^{13.2} M_{\odot}$ we have a BH mass $M_{\bullet} \approx 2 \times 10^{9} M_{\odot}$ while they have $M_{\bullet} \approx 8 \times 10^{9} M_{\odot}$. Recall that we obtain the relation of Eq. (2) as a self-consistent output of the equations in Appendix A, that follow the details of the building up of the central BH mass.

In summary, the comparison of our results with those of Wyithe \& Loeb (2003) and Mahmood et al. (2004) is complex, because of quite different assumptions. Despite of these, similar QSO LFs are produced. At high redshift their lower $t_{\mathrm{vis}} \lesssim 10^{7}$ yr and formation rates of DM halos are compensated by: (i) neglecting the time needed to build up the galactic structure and (ii) assuming a relationship $M_{\bullet}-M_{\text {vir }}$ that favors larger BH masses. The difference emerges quite clearly at redshifts around $7-8$, where we predict a dramatic drop off of the LF at high luminosity, because of the delay (see $\S 4.1$ ), whereas these authors obtain number densities larger than ours by a factor of 10 .

Clear differences are also present on the host galaxy side. In our model the time $\Delta t_{\text {peak }}$ is a very important and active phase, within which the massive host galaxies are forming stars at rates around $10^{2}-10^{3} M_{\odot} \mathrm{yr}^{-1}$ (cf. Fig. 1) and the central $\mathrm{BH}$ is growing. This phase is directly explored by sub-mm surveys and by the observations of the X-ray emission from the growing QSOs detected by Alexander et al. (2005).

More recently, several authors tried to follow the evolution of both QSOs and host galaxies using results from numerical simulations, in which the effects of SN explosions and winds from the central active nucleus are taken into account (see Hopkins et al. 2006 and references therein). These authors took an approach complementary to that presented in this paper. Instead of solving equations describing the basic physical processes, they simulated many realizations of collisions between two stable, isolated disk galaxies endowed with a central supermassive BH. A two-phase interstellar medium was used to describe star formation and SN feedback (Springel \& Hernquist 2003). The accretion onto the BHs was estimated from the local gas density and sound speed, and limited to the Eddington rate. They assumed that $5 \%$ of the bolometric luminosity derived from accretion is transferred to the surrounding gas, as thermal energy. They found that during a major merger the central BHs are fed with enough gas to yield a luminosity depending exponentially on time. Their approach includes also estimates of absorption by dust. The energy injected by the QSO in the interstellar medium is enough to unbind the gas itself on a time scale $\Delta t_{\mathrm{vis}} \sim 10^{9} \mathrm{yr}$, within which the luminosity varies from about $10^{-3} L_{\text {peak }}$ to a maximum $L_{\text {peak }}$, with a weak dependence on $L_{\text {peak }}$ [cf. Eq. (7) of Hopkins et al. 2006]. Note that these simulations do recover the transition from obscured inflow/BH growth to QSO-driven blowout, as we have simply modeled from the relevant accretion/feedback equations.

The time scale $\Delta t_{\text {vis }}$ for the growth of the BHs derived from numerical simulations of low-redshift galaxy mergers at large halo mass has the same meaning as our time $\Delta t_{\text {peak }}$ when the maximum luminosity is attained. However, for large masses and high redshifts the two timescales have quite different values, $\Delta t_{\text {vis }} \approx 10^{9} \mathrm{yr} \gtrsim \Delta t_{\text {peak }} \approx 0.2-0.5$ Gyr for $z_{\text {vir }} \geq 4$. The long time before the peak luminosity is reached has strong implications at high redshifts. In particular, $\Delta t_{\text {vis }} \sim t_{H}$ for $z \gtrsim 4$. Thus the model of two colliding discs takes too long to form supermassive BHs at high $z$ (recall that the discs themselves need at least a time $R_{\text {vir }} / V_{\text {vir }}$ 


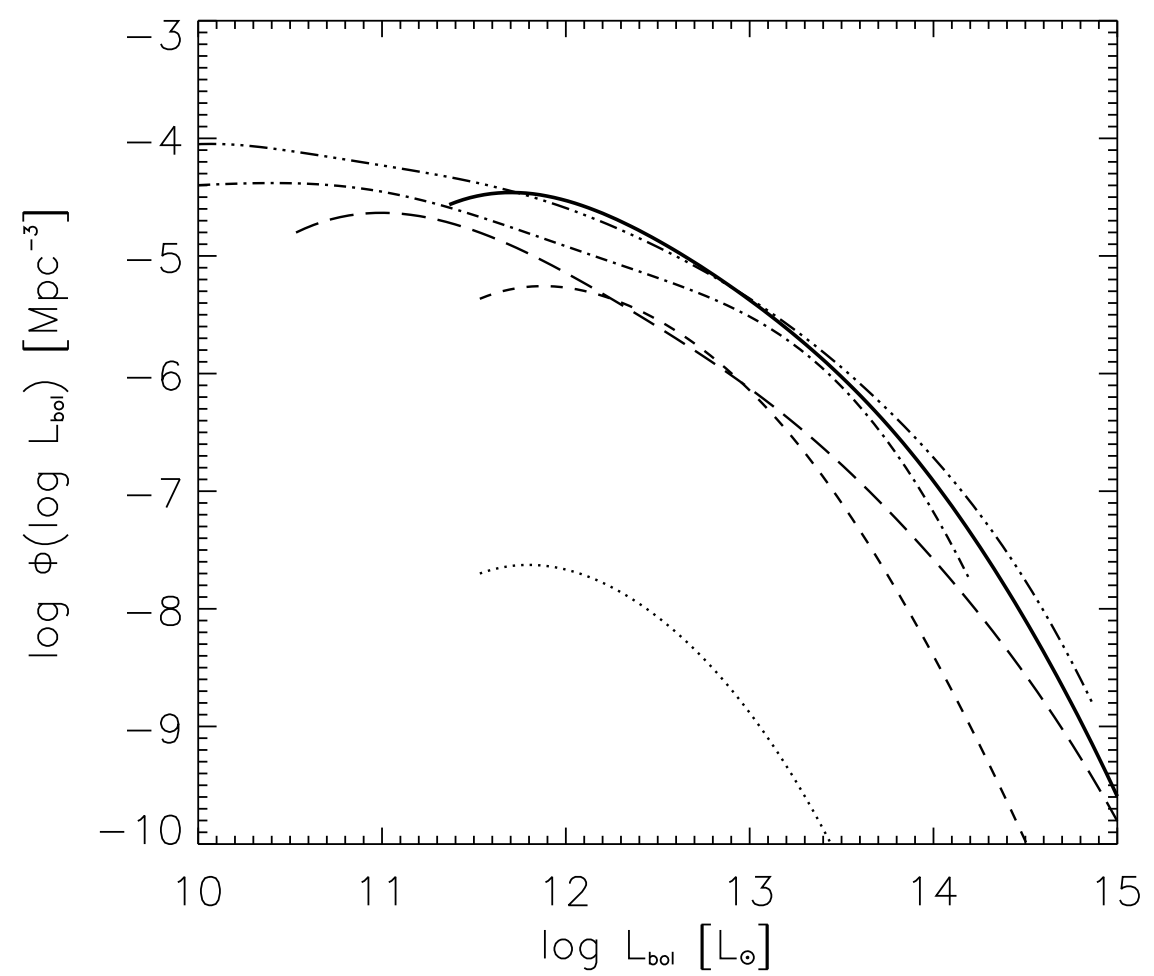

FIG. 8.- Bolometric QSO LFs predicted by our model at redshifts $z \approx 8$ (dotted line), $z \approx 6$ (short dashed line), $z \approx 5$ (long dashed line), $z \approx 3$ (solid line), $z \approx 2$ (triple dot-dashed line), and $z \approx 1.5$ (dot-dashed line).

to set up). Therefore the extension of the model at $z \gtrsim 2$ is quite complex, as discussed by Hopkins et al. (2005) in their $\$ 3.2$. Moreover, it is likely that several properties inferred from this kind of low-redshift merger simulations cannot be extrapolated to high redshifts, where the very idea of binary galaxy mergers likely breaks down. However, it is interesting to note that at lower redshifts, $z \lesssim 1.5$, the two times $\Delta t_{\text {vis }}$ and $\Delta t_{\text {peak }}$ are comparable. Our model is not aimed at following the evolution of QSOs at $z \lesssim 1$. This later phase is better described by models that include 'interactions' (minor mergers, fly-bys, disk instabilities, etc.) among galaxies as triggers of nuclear activity (e.g., Kauffmann \& Haehnelt 2000; Cavaliere \& Vittorini 2000; Menci et al. 2003).

\section{DISCUSSION AND CONCLUSIONS}

In their thoughtful review paper, Brandt \& Hasinger (2005) list some 'key outstanding problems' of AGN astrophysics that need investigation. These include: the detailed cosmic history of supermassive BH accretion; the nature of AGN activity in young, forming galaxies, and the connection between supermassive BH growth and star formation in sub-mm galaxies.

In this paper we address these issues in the framework of the anti-hierarchical baryon collapse (ABC) scenario developed by Granato et al. (2004). We have shown that the condensation of baryons within DM halos both in stars and in BHs can be described with a simple physical model, whose main equations are listed in Appendix A. The model yields the time dependence of the SFR, of the accretion rate $\dot{M}_{\bullet}$ onto the central BH, of the total mass in stars $M_{\star}$ and of the final BH mass $M_{\bullet}$, for any given halo mass and virialization epoch. The GRASIL code then provides the SED from X-ray to radio bands of evolving stellar populations (successfully reproducing the epoch-dependent galaxy LFs in different spectral bands, as well as a variety of relationships among photometric, dynamical and chemical properties, as shown in previous papers; see Table A2), while observationally determined bolometric corrections allow us to convert the accretion rates onto supermassive BHs into luminosities in optical and X-ray bands.

In the $\mathrm{ABC}$ scenario, the growth rate of BHs is proportional to the SFR, and the latter is more effectively slowed down by SN feedback in smaller halos, resulting in a relatively more efficient growth of more massive BHs, especially at higher redshifts. Both star formation and $\mathrm{BH}$ growth in massive halos are stopped by the feedback from the active nucleus as soon as it becomes powerful enough to sweep out the residual interstellar medium.

The BH accretion occurs in host galaxies with very intense star formation, and is heavily dust obscured for the majority of its duration; however, most of the final $\mathrm{BH}$ mass is gained during the last $1-2 e$-folding times in unobscured conditions, as demanded e.g. by the Soltan (1982) argument and by the analysis of Yu \& Tremaine (2002). For massive objects, the growth phase lasts 15-20e-folding times of Eddington-limited accretion, but the AGNs are detectable by current hard X-ray surveys only in the last several $e$-folding times, i.e. have hard X-ray visibility times of $\sim 3 \times 10^{8}$ yr. Interestingly, Borys et al. (2005) estimate that the $\mathrm{BH}$ masses associated to the X-ray emitting AGNs detected in sub-mm galaxies are, on average, $\sim 50$ times lower than those associated to local spheroidal galaxies with similar stellar masses, as expected if they are about $4 e$-folding times before the maximum, for Eddington-limited accretion. At still earlier times, sub-mm galaxies are expected to host intrinsically weak and highly obscured nuclei, undetectable with current X-ray telescopes. 
The model accounts for the hard X-ray AGN LFs at various redshifts. A crucial ingredient, to this end, is the rapid quenching of the accretion rate onto the biggest BHs, predicted by the model as the consequence of the AGN feedback which sweeps out the interstellar medium.

The optical (B-band) visibility time is shorter than that for the more penetrating hard X-rays. The observed epoch-dependent B-band LFs are accurately reproduced for a $\Delta t_{\text {vis }}$ of order of the $e$-folding time, indicating that only when the AGN is approaching its maximum luminosity it can clear up the surrounding region. The redshift dependence of the space density of optically bright QSOs is controlled, in the ABC scenario, by two competing factors. On one side, according to the hierarchical clustering paradigm, the formation rate of very massive halos hosting them is increasing rapidly with decreasing redshift. On the other side, the $\mathrm{BH}$ to host halo mass ratio decreases with decreasing redshift, so that at low redshifts relatively more massive hosts are required for a given $\mathrm{BH}$ mass. But the galactic halo mass function sinks down exponentially for large halo masses, and is actually cut off at $M_{\mathrm{vir}} \approx 2 \times 10^{13} M_{\odot}$. In our model, the first factor dominates for $z \gtrsim 2.5$ and the second dominates at lower $z$, thus accounting for the increase in the bright QSO space density with decreasing $z$ down to $z \approx 2.5$, as well as for the subsequent decrease.

The redshift dependence of the bolometric QSO LF is illustrated by Fig. 8 It is broadly reminiscent of luminosity evolution, but with significant deviations from that simple description particularly at high luminosities. We note in particular the flattening at high luminosity around $z \approx 5$, borne out by SDSS data (Fan et al. 2001) and the very sharp drop (by about 2 orders of magnitude) of the QSO LF between $z \approx 6$ and $z \approx 8$, due to the dearth of massive halos at high redshifts.

As stated in $\S 3.1$ and 4.1 our model requires a mild variation of the Eddington ratio $\lambda$ with redshift. This may be a matter of concern, since the value of $\lambda$ is determined mainly by the environmental conditions very close to the supermassive BHs. The main motivation for resorting to super-Eddington accretion at high $z$ is to account for the observed space density of very luminous QSOs at $z \gtrsim 5$ (an acceptable fit to lower redshift data can be obtained keeping $\lambda \approx 1$ ). Since, as indicated by Eq. (5), QSOs seen at the cosmic time $t$ are associated to halos virializing at the earlier time $t_{\mathrm{vir}} \lesssim t-\Delta t_{\text {peak }}$, and the density of such halos drops with increasing $\Delta t_{\text {peak }}$ at high $z$, to have a sufficient number of massive enough BHs at $z \gtrsim 5$ we need them to grow faster than implied by Eddington-limited accretion. The values of $\lambda$ mentioned in $\S 3.1$ provide the necessary shortening of $\Delta t_{\text {peak }}$ and also give high enough luminosities without resorting to too large $\mathrm{BH}$ masses. On the other hand, if we want to preserve the relationships between the properties of the QSO and of the host galaxy, $\Delta t_{\text {peak }}$ must be long enough to allow the formation of a sufficiently massive stellar component.

The redshift dependence of $\lambda$ may thus be seen as a way to parameterize aspects of the physics of the accretion/emission process not properly taken into account by our simple model. For example, a major improvement could be obtained by taking into account the results of the photon trapping theory (e.g., Blandford 2004). According to the latter, radiative emissions close to Eddington can be originated by strongly super-Eddington accretion rates (e.g., those allowed in our model by viscous accretion). This process seems promising because it may naturally allow short delay times at high redshift even with an Eddington-limited radiative output. However, details of the mechanism are still uncertain and additional work is needed to quantitatively include it in our model. Non-radiative accretion may also speed up the BH growth. On top of that, the values of the Eddington ratio could have a substantial scatter due to different environmental conditions around the supermassive BHs. As a result, the samples of high redshift QSOs may be biased towards higher Eddington ratios.

We note that since luminous high- $z$ QSOs are associated to very massive halos, the model implies that their clustering properties are similar to those of massive spheroidal galaxies and of the bright sub-mm galaxies detected by the SCUBA surveys, consistent with the results by Porciani et al. (2004) and by Croom et al. (2005). This issue will be investigated in a future paper.

During the relatively short phase when massive galaxies are in the process of expelling their gas and dust, their nuclei should appear as powerful X-ray sources, $L_{X} \gtrsim 10^{45} \mathrm{erg} \mathrm{s}^{-1}$, but still somewhat obscured in the optical band; they thus may resemble Type 2 QSOs.

Spectroscopic studies, aimed at determining velocity, amount and chemical abundances of the gas ejected during the QSO phase, would be extremely informative. In fact, the model predicts that at early cosmic times large amounts of metal-enriched gas were evacuated from the galaxy halos to the intergalactic medium.

We warmly thank S. Bressan, A. Celotti, and M. Cirasuolo for helpful discussions, and the anonymous referee for a careful and perceptive report, rich of illuminating comments. This work is supported by grants from ASI, INAF and MIUR.

\section{REFERENCES}

Alexander D.M., Bauer, F.E., Chapman, S.C., Smail, I., Blain, A.W., Brandt, W.N., \& Ivison, R.J., 2005, ApJ, 632, 736

Bardeen J., Bond J., Kaiser N. \& Szalay A., 1986, ApJ, 304, 15

Barger, A.J., Cowie, L.L., Mushotzky, R.F., Yang, Y., Wang, W.-H., Steffen, A.T., \& Capak, P., 2005, AJ, 129, 578

Baugh, C.M., Lacey, C.G., Frenk, C.S., Granato, G.L., Silva, L., Bressan, A. Benson, A. J., \& Cole, S., 2005, MNRAS, 356, 1191

Bennett, C.L., et al., 2003, ApJS, 148, 1

Bernardi, M., et al., 1998, ApJ, 508, L143

Blandford, R.D., 2004, in Coevolution of Black Holes and Galaxies, ed. L.C. Ho (Cambridge: Cambridge Univ. Press), 153

Bond, J.R., Cole, S., Efstathiou, G., \& Kaiser, N., 1991, ApJ, 379, 440

Borys C., Smail I., Chapman S.C., Blain A.W., Alexander D.M. \& Ivison R.J., 2005, ApJ, 635, 853

Bouwens, R., \& Illingworth, G., 2006, NewAR, 50, 152

Bower, R.G., Lucey, J.R.. Ellis, R.S., 1992, MNRAS, 254, 589

Brandt, W.N., \& Hasinger, G., 2005, ARA\&A, 43, 827

Brandt, W.N., \& Gallagher, S.C., 2000, NewAR, 44, 461
Bromley, J.M., Somerville, R.S., \& Fabian, A.C., 2004, MNRAS, 350, 456

Bundy, K., Ellis, R.S., Conselice, C.J., 2005, ApJ, 625, 621

Caldwell, N., Rose, J.A., \& Concannon, K.D., 2003, AJ, 125, 2891

Caputi, K.I., McLure, R.J., Dunlop, J.S., Cirasuolo, M., \& Schael, A.M., 2006, MNRAS, 366, 609

Cavaliere, A., \& Vittorini, V., 2000, ApJ, 543, 599

Chapman, S.C., Blain, A.W., Smail, I., \& Ivison, R.J., 2005, ApJ, 622, 772

Chapman, S.C., Blain, A.W., Ivison, R.J., \& Smail, I., 2003, Nature, 422, 695

Chartas, G., Brandt, W.N., \& Gallagher, S.C., 2003, ApJ, 595, 85

Cimatti, A., et al., 2002, A\&A, 392, 395

Cirasuolo M., Shankar F., Granato G.L., De Zotti G. \& Danese L., 2005, ApJ, 629,816

Cox, T.J., Di Matteo, T., Hernquist, L., Hopkins, P.F., Robertson, B., \& Springel, V., 2006, ApJL, submitted [preprint astro-ph/0504156

Cristiani S., et al., 2004, ApJ, 600, L119

Croom, S.M., et al., 2005, MNRAS, 356, 415

Croom S.M. et al., 2004, MNRAS, 349, 1397

Croton, D.J., et al., 2006, MNRAS, 365, 11 
D' Odorico, V., Cristiani, S., Romano, D., Granato, G.L., \& Danese, L., 2004, MNRAS, 351, 976

Di Matteo, T., Springel, V., \& Hernquist, L., 2005, Nature, 433, 604

Dressler, A., et al., 1987, ApJ, 313, 42

Dunlop, J.S., McLure, R.J., Kukula, M.J., Baum, S.A., O' Dea, C.P., \& Hughes, D.H., 2003, MNRAS, 340, 1095

Ellis, R.S., et al., 1997, ApJ, 483, 582

Elvis, M., et al., 1994, ApJS, 95, 1

Fan, X., et al., 2006, AJ, 131, 1203

Fan, X., et al., 2004, ApJ, 128, 515

Fan, X. et al., 2001, AJ, 121, 54

Ferrarese, L., \& Merritt, D., 2000, ApJ, 539, L9

Floyd, D.J.E., et al. 2004, MNRAS, 355, 196

Fontana, A., et al., 2004, A\&A, 424, 23

Gebhardt, K., et al., 2000, ApJ, 539, L13

Gebhardt, K., et al., 2001, AJ, 122, 2469

Gerhard, O., Kronawitter, A., Saglia, R.P., \& Bender, R., 2001, AJ, 121, 1936

Giacconi, R., 1985, Bulletin of the AAS, 17, 888

Granato, G.L., Silva, L., Lapi, A., Shankar, F., De Zotti, G., \& Danese, L., 2006, MNRAS, 368, L72

Granato G.L., De Zotti G., Silva L., Bressan A. \& Danese L., 2004, ApJ, 600, 580

Granato G.L., Silva L., Monaco P., Panuzzo P., Salucci P., De Zotti G. \& Danese L., 2001, MNRAS, 324, 757

Greene, J.E., \& Ho, L.C., 2006, ApJ, 641, L21

Greve, T.R., Bertoldi, F., Smail, I., Neri, R., \& Chapman, S.C., 2005, MNRAS, 359, 1165

Haehnelt, M.G., \& Rees, M.J., 1993, MNRAS, 263, 168

Haiman Z. \& Loeb A., 1998, ApJ, 503, 505

Holden, B.P., et al., 2005, ApJ, 620, L83

Hopkins, P.F., Hernquist, L., Cox, T.J., Di Matteo, T., Robertson, B., \& Springel, V., 2006a, ApJS, 163, 1

Hopkins, P.F., Hernquist, L., Cox, T.J., Robertson, B., Di Matteo, T., \& Springel, V., 2006b, ApJ, 639, 700

Hunt, M.P., Steidel, C.C., Adelberger, K.L., \& Shapley, A.E., 2004, ApJ, 605, 625

Jedrzejewski, R.I., Davies, R.L., Illingworth, G.D., 1987, AJ, 94, 1508

Jørgensen, I., Franx, M, Hjorth, J., van Dokkum, P.G., 1999, MNRAS, 308, 833

Kashikawa, N., Takata, T., Ohyama, Y., \& Yoshida, M., 2003, AJ, 125, 53

Kauffmann, G., et al., 2003, MNRAS, 346, 1055

Kauffmann, G., \& Haehnelt, M., 2000, MNRAS, 311, 576

Kawakatu, N., \& Umemura, M.. 2002, MNRAS, 329, 572

Kennefick, J.D., Djorgovski, S.G., \& de Carvalho, R.R., 1995, AJ, 110, 2553

King, A.R., \& Pounds, K.A., 2003, MNRAS, 345, 657

Kitayama, T., \& Suto, Y., 1996, MNRAS, 280, 638

Kleinheinrich, M. et al., 2005, A\&A, 439, 513

Kochanek, C.S., \& White, M., 2001, ApJ, 559, 531

Kodama, T., et al., 1998, A\&A, 334, 99

Kollmeier, J.A., et al., 2006, ApJ, in press [preprint astro-ph/0508657

Koopmans, L.V.E., Treu, T., Bolton, A.S., Burles, S., \& Moustakas, L.A. 2006, ApJ, submitted [preprint astro-ph/0601628

Kormendy, J., \& Richstone, D., 199J, ARA\&A, 33, 581

Kronawitter, A., Saglia, R.P., Gerhard, O., \& Bender, R. 2000, A\&AS, 144, 53

Lacey, C., \& Cole, S., 1993, MNRAS, 262, 627

La Franca F. et al., 2005, ApJ, 635, 864

Lapi A., Cavaliere A. \& Menci N., 2005, ApJ, 619, 60

Magliocchetti, M., \& Porciani, C., 2003, MNRAS, 346, 186

Magorrian, J., et al., 1998, AJ, 115, 2285

Mahmood A., Devriendt J.E.G. \& Silk J., 2005, MNRAS, 359, 1363

Marconi A., Risaliti G., Gilli R., Hunt L. K., Maiolino R. \& Salvati M., 2004, MNRAS, 351, 169

Marconi, A., \& Hunt, L.K., 2003, ApJ, 589, L21

Mathez, G., 1978, A\&A, 68, 17

McLure, R.J., \& Dunlop, J.S., 2004, MNRAS, 352, 1390

Menci, N., Cavaliere, A., Fontana, A., Giallongo, E., Poli, F., \& Vittorini, V., 2003, ApJ, 587, L63

Mo, H.J., \& Mao, S., 2004, MNRAS, 353, 829
Murray, N., Chiang, J., Grossman, S.A., \& Voit, G.M. 1995, ApJ, 451, 498 Naab, T., Khochfar, S., \& Burkert, A., 2006, ApJ, 636, L81

Nelan, J.E., et al., 2005, ApJ, 632, 137

Onken, C.A., et al., 2004, ApJ, 615, 645

Pei Y. C., 1995, ApJ, 438, 623

Porciani, C., Magliocchetti, M., \& Norberg, P., 2004, MNRAS, 355, 1010

Press, W.H., \& Schechter, P., 1974, ApJ, 187, 425

Richards, G.T., et al., 2006, AJ, 131, 2766

Richards, G.T., et al., 2005, MNRAS, 360, 839

Romano, D., Silva, L., Matteucci, F., \& Danese, L., 2002, MNRAS, 334, 444

Salucci, P., Ratnam, C., Monaco, P., \& Danese, L. 2000, MNRAS, 317, 488

Sandage, A.. \& Visvanathan, N. 1978, ApJ, 223, 707

Sanders, D.B., Soifer, B.T., Elias, J.H., Madore, B.F., Matthews, K., Neugebauer, G., \& Scoville, N.Z., 1988, ApJ, 325, 74

Sasaki, S., 1994, PASJ, 46, 427

Schmidt, M., \& Green, R.F., 1986, ApJ, 305, 68

Shankar F., Lapi A., Salucci P., G. de Zotti \& Danese L., 2006, ApJ, 643, 14

Shankar F., Salucci P., G.L. Granato, G. de Zotti \& L. Danese, 2004, MNRAS, 354, 1020

Sheth, R.K., et al., 2003, ApJ, 594, 225

Sheth, R.K., \& Tormen, G., 2002, MNRAS, 329, 61

Sheth, R.K., \& Tormen, G., 1999, MNRAS, 308, 119

Silva, L., De Zotti, G., Granato, G.L., Maiolino, R., \& Danese, L. 2005, MNRAS, 357, 1295

Silva, L., Granato, G.L., Bressan, A., \& Danese, L. 1998, ApJ, 509, 103

Small, T.A., \& Blandford, R.D., 1992, MNRAS, 259, 725

Soltan, A., 1982, MNRAS, 200, 115

Somerville, R.S., et al., 2004, ApJ, 600, L135

Spergel, D.N., et al., 2006, ApJ, submitted [preprint astro-ph/0603449

Springel, V., et al., 2005, Nature, 435, 629

Springel, V., \& Hernquist, L., 2003, MNRAS, 339, 312

Srianand, R \& Petitjean, P, 2000, A\&A, 357, 414

Strateva, I.V., et al. 2005, AJ, 130, 387

Sugiyama, N., 1995, ApJS, 100, 281

Sutherland, R.S., \& Dopita, M.A., 1993, ApJS, 88, 253

Tecza, M., et al., 2004, ApJ, 605, L109

Terlevich, A.I., \& Forbes, D.A., 2002, MNRAS, 330, 547

Thomas, D., Maraston, C., Bender, R., de Oliveira, C.M., 2005, ApJ, 621, 673

Tozzi, P., et al. 2001, ApJ, 562, 42

Trager, S.C., Faber, S.M., Worthey, G., González, J.J., 2000, AJ, 119, 1645

Tremaine, S., et al. 2002, ApJ, 574, 740

Treu, T., Stiavelli, M., Casertano, S., Møller, P., \& Bertin, G., 2002, ApJ, 564, L13

Ueda Y., Akiyama M., Ohta K. \& Miyaji T., 2003, ApJ, 598, 886

Vale, A., \& Ostriker, J.P., 2006, MNRAS, submitted [preprint astro-ph/0511816

Vale, A., \& Ustriker, J.P., 2004, MNRAS, 353, 189

Valluri, M., Ferrarese, L., Merritt, D., \& Joseph, C.J., 2005, ApJ, 628, 137

van den Bosch, F.C., Tormen, G., \& Giocoli, C., 2005, MNRAS, 359, 1029

van der Wel, A., Franx, M., van Dokkum, P.G., \& Rix, H.-W., 2004, ApJ, 601, L5

Vestergaard, M., \& Peterson, B.M., 2006, ApJ, 641, 689

Vestergaard, M., 2004, ApJ, 601, 676

Vignali, C., Brandt, W.N., \& Schneider, D.P. 2003, AJ, 125, 433

Voit, G.M., Rev. Mod. Phys., in press [preprint astro-ph/0410173

Warner, C., Hamann, F., \& Dietrich, M., 2004, ApJ, 608, 136

Wechsler, R.H., Bullock, J.S., Primack, J.R., Kravtsov, A.V., Dekel, A., 2002, ApJ, 568, 52

Wilkes, B., 1994, in ASP Conf. Ser. 61, Astronomical Data Analysis Software and Systems III, eds. D.R. Crabtree, R.J. Hanisch and J. Barnes, p. 423

Wolf, C., Wisotzki, L., Borch, A., Dye, S., Kleinheinrich, M., \& Meisenheimer, K., 2003, A\&A, 408, 499

Wyithe, J.S.B. \& Loeb, A., 2003, ApJ, 595, 614

Yu, Q. \& Lu, Y., 2004, ApJ, 602, 603

Yu, Q., \& Tremaine, S., 2002, MNRAS, 335, 965

Zhao, D.H., Mo, H.J., Jing, Y.P., Börner, G., 2003a, MNRAS, 339, 12

Zhao, D.H., Jing, Y.P., Mo, H.J., \& Börner, G., 2003b, ApJ, 597, L9

\section{APPENDIX}

\section{BARYONIC PHYSICS IN OUR GALAXY EVOLUTION MODEL}

In this Appendix we provide an overview of the physical model by Granato et al. (2004), recalling the basic equations and parameters that control the evolution of the baryonic component.

The baryonic content of a given DM halo with mass $M_{\text {vir }}$ is partitioned in three gaseous phases: a hot diffuse medium with mass $M_{\text {inf }}$ infalling and/or cooling toward the center; cold gas with mass $M_{\text {cold }}$ condensing into stars; low-angular momentum gas with mass $M_{\text {res }}$ stored in a reservoir around the central supermassive $\mathrm{BH}$, and eventually viscously accreting onto it. In addition, two condensed phases are present, namely, stars with a total mass $M_{\star}$ and the $\mathrm{BH}$, with mass $M_{\bullet}$.

The evolution of the baryonic content is described by the system of differential equations

$$
\begin{aligned}
& \dot{M}_{\mathrm{inf}}=-\dot{M}_{\mathrm{cond}}-\dot{M}_{\mathrm{inf}}^{Q S O}, \\
& \dot{M}_{\mathrm{cold}}=\dot{M}_{\mathrm{cond}}-\dot{M}_{\star}-\dot{M}_{\mathrm{res}}-\dot{M}_{\mathrm{cold}}^{S N}-\dot{M}_{\mathrm{cold}}^{Q S O},
\end{aligned}
$$


TABLE A1

MODEL PARAMETERS

\begin{tabular}{lll}
\hline \hline Parameter & Value & \multicolumn{1}{c}{ Short description } \\
\hline $\mathcal{C}$ & 7 & clumping factor \\
$\epsilon_{S N}$ & 0.05 & strength of SN feedback \\
$\alpha_{R D}$ & 2.5 & strength of radiation drag \\
$\tau_{0}$ & 1 & zero-point of optical depth \\
$M_{\bullet}^{\text {seed }}$ & $10^{2} M_{\odot}$ & mass of BH seed \\
$k_{\text {accr }}$ & $10^{-2}$ & strength of viscous accretion \\
$\lambda$ & $0.8-4$ & Eddington ratio* \\
$\eta$ & 0.15 & radiative efficiency \\
$\epsilon_{Q S O}$ & 1.3 & strength of QSO feedback
\end{tabular}

Note. $-{ }^{*}$ We let the maximum allowed Eddington ratio $\lambda$ to depend on the redshift as: $\lambda=4$ for $z \geq 6, \lambda=3$ for $5 \lesssim z \lesssim$ $6, \lambda=1.7$ for $3 \lesssim z \lesssim 5, \lambda=1$ for $2 \lesssim z \lesssim 3$, and $\lambda=0.8$ for $1.5 \lesssim z \lesssim 2$; the empirical fit $\lambda(z) \approx-1.15+0.75(1+z)$ works as well in the redshift range $1.5 \lesssim z \lesssim 6$.

$$
\dot{M}_{\text {res }}=\dot{M}_{\text {inflow }}-\dot{M}_{\bullet},
$$

where $\dot{M}_{\text {cond }}$ is defined by Eq. (A2). At the virialization redshift $z_{\text {vir }}$ we set $M_{\text {inf }} \approx M_{\text {vir }} / 6$ corresponding to the cosmic baryon to DM ratio and featuring a primordial chemical composition, $M_{\bullet} \approx M_{\bullet}^{\text {seed }}, M_{\bullet}^{\text {seed }}$ being a BH seed mass originated by some process in the early universe, and the other baryonic components to zero.

The hot gas condenses/cools down at the rate

$$
\dot{M}_{\text {cond }}=\frac{M_{\text {inf }}}{\max \left[t_{\text {cool }}, t_{\text {dyn }}\right]},
$$

determined by the dynamical and cooling timescales $t_{\mathrm{dyn}}$ and $t_{\mathrm{cool}}$ at the virial radius. The latter includes the appropriate cooling function (Sutherland \& Dopita 1993) and allows for a clumping factor $\mathcal{C}$ in the baryonic component. Since at high redshifts major mergers between massive halos are very frequent, we neglect here the effect of the angular momentum, since it is lost by dynamical friction through mergers of mass clouds $M_{c}$ on time scale $t_{\mathrm{DF}} \approx 0.2(\xi / \ln \xi) t_{\mathrm{dyn}}$, where $\xi=M_{\mathrm{vir}} / M_{c}$ (see e.g. Mo \& Mao 2004); major mergers imply $\xi \sim$ a few. In this context it is also worth noticing that the gas that collapses and cools enough to form stars is only a fraction of less than $30 \%$ of that associated to the virialized halo (cf. Fig. 11).

The cold gas turns into stars at the rate

$$
\dot{M}_{\star}=\int \frac{\mathrm{d} M_{\text {cold }}}{\max \left[t_{\text {cool }}, t_{\text {dyn }}\right]},
$$

where now $t_{\text {cool }}$ and $t_{\mathrm{dyn}}$ refer to cooling and dynamical time, respectively, in the radial mass shell of mass $\mathrm{d} M_{\text {cold }}$.

The energy feedback from Type-II SNae removes gas from the cold phase at the rate

$$
\dot{M}_{\text {cold }}^{S N} \approx \epsilon_{S N}\left(\frac{V_{\text {vir }}}{500 \mathrm{~km} / \mathrm{s}}\right)^{-2} \dot{M}_{\star},
$$

where the efficiency $\epsilon_{S N}$ is a parameter of the model. The amount of cold mass removed is proportional to the number of SN explosions (assuming an average energy of around $10^{51}$ ergs per SN) hence to the SFR, and inversely proportional to the depth of the halo potential well.

The stellar radiation drag (see Kawakatu \& Umemura 2002) triggers the inflow of cold gas into a reservoir of low-angular momentum around the central supermassive $\mathrm{BH}$, at the rate

$$
\dot{M}_{\text {inflow }} \approx \alpha_{R D} \times 10^{-3} \dot{M}_{\star}\left(1-e^{-\tau}\right) M_{\odot} \mathrm{yr}^{-1},
$$

where the effective optical depth is given by

$$
\tau=\tau_{0}\left(\frac{Z}{Z_{\odot}}\right)\left(\frac{M_{\text {cold }}}{10^{12} M_{\odot}}\right)\left(\frac{M_{\text {vir }}}{10^{13} M_{\odot}}\right)^{-2 / 3} .
$$

The metallicity $Z(t)$ is computed from the code self-consistently, while the coefficients $\alpha_{R D}$ and $\tau_{0}$ are parameters (see Granato et al. (2004) for further details).

The viscous time $t_{\mathrm{visc}}=\mathrm{Re}_{\text {crit }} t_{\mathrm{dyn}}^{\mathrm{BH}+\mathrm{res}}$ for the accretion from the reservoir to the $\mathrm{BH}$ is related to the dynamical time $t_{\mathrm{dyn}}^{\mathrm{BH}+\mathrm{res}}$ of the system $\mathrm{BH}+$ reservoir, and to the critical Reynolds number $\mathrm{Re}_{\text {crit }} \sim 10^{2}-10^{3}$ (see Granato et al. 2004 for details). Thus the gas in the reservoir accretes onto the supermassive $\mathrm{BH}$ at the rate

$$
\dot{M}_{\bullet}^{\text {visc }} \approx 5 \times 10^{3} k_{\text {accr }}\left(\frac{V_{\text {vir }}}{500 \mathrm{~km} \mathrm{~s}^{-1}}\right)^{3}\left(\frac{M_{\text {res }}}{M_{\bullet}}\right)^{3 / 2}\left(1+\frac{M_{\bullet}}{M_{\text {res }}}\right)^{1 / 2} M_{\odot} \mathrm{yr}^{-1}
$$


TABLE A2

OVERVIEW OF MOdel RESults

\begin{tabular}{lll}
\hline \hline Property or Statistics & \multicolumn{1}{c}{ External Inputs ${ }^{\star}$} & \multicolumn{1}{c}{ Reference } \\
\hline & & \\
$Q S O s$ and $B H s$ & & \\
$M \bullet-M_{\text {vir }}$ & no add & Fig. 2 (left) \\
$\Delta t_{\text {peak }}-M_{\text {vir }}$ & no add & Fig. 2 (right) \\
Opt. LFs $(z)$ & $\Delta t_{\text {vis }} \approx 5 \times 10^{7} \mathrm{yr}, \Delta \log M \bullet \approx 0.3$ & Figs. 3-6 \\
X-ray LFs $(z)$ & $\Delta t_{\text {vis }} \approx 3 \times 10^{8} \mathrm{yr}$ & Figs. 5-6 (right) \\
BH mass function & no add & Fig. 7 \\
$M \bullet-\sigma$ & no add & Fig. 6 of G04 \\
& & \\
Spheroidal Galaxies & & \\
VDF & & \\
Faber-Jackson & $\sigma / V_{\text {vir }}$ & Fig. 1 of C05 \\
LF $(z)$ & IMF, SEDs (GRASIL) & Fig. 2 of C05 \\
Metal abundances & IMF, chemical yields & Fig. 10 of G04 \\
K-band counts & IMF, dust modeling (GRASIL) & Fig. 9 of G04 \\
$850 \mu$ m counts & IMF, dust modeling (GRASIL) & Fig. 12 of G05 \\
EROs & IMF, dust modeling (GRASIL) & Fig. 6 of S05 \\
& &
\end{tabular}

Note. $-{ }^{\star}$ Input quantities not included in Table A1. G04: Granato et al. (2004), C05: Cirasuolo et al. (2005), S05: Silva et al. (2005).

Again, the coefficient $k_{\text {accr }}$ is a model parameter. For the plausible range of $10^{-4} \lesssim k_{\text {accr }} \lesssim 10^{-2}$, the accretion rate $\dot{M}_{\bullet}^{\text {visc }}$ easily exceeds the Eddington rate for high redshifts and massive halos. In this situation we may have: (i) very rapid growth of the central BH in a dynamical timescale, followed by a slower accretion when less mass is available in the reservoir; (ii) BH growth limited by radiation pressure, followed by strong mass outflows $\dot{M}_{w} \sim \dot{M}_{\text {Edd }}$ (e.g., Small \& Blandford 1992; King \& Pounds 2003; Begelmann 2004). We select the latter option and the actual accretion rate is set to

$$
\dot{M}_{\bullet}=\min \left[\dot{M}_{\bullet}^{\text {visc }}, \lambda \dot{M}_{\bullet}^{\text {Edd }}\right],
$$

where

$$
\dot{M}_{\bullet}^{\text {Edd }} \approx 1.3\left(\frac{M_{\bullet}}{10^{8} M_{\odot}}\right) M_{\odot} \mathrm{yr}^{-1}
$$

is the Eddington rate and $\lambda$ is the maximum allowed Eddington ratio. We let the maximum allowed Eddington ratio $\lambda$ to depend on the redshift as: $\lambda=4$ for $z \gtrsim 6, \lambda=3$ for $5 \lesssim z \lesssim 6, \lambda=1.7$ for $3 \lesssim z \lesssim 5, \lambda=1$ for $2 \lesssim z \lesssim 3$, and $\lambda=0.8$ for $1.5 \lesssim z \lesssim 2$. The empirical fit $\lambda(z) \approx-1.15+0.75(1+z)$ works as well in the redshift range $1.5 \lesssim z \lesssim 6$, see also Table A1.

In the presence of Eddington-limited or super-Eddington accretion, QSO-driven outflows are expected in the form of winds that can form just above the disc by a combination of radiation and gas pressure. Large mass outflows have been confirmed by X-ray observations of BAL QSOs (Brandt \& Gallagher 2000; Chartas et al. 2003). Following the model by Murray et al. (1995) the asymptotic speed and the mass outflow rates are estimated as $v_{w} \approx 0.06 c\left(r_{w} / 10^{16} \mathrm{~cm}\right)^{-1 / 2}\left(\dot{M}_{\bullet} / M_{\odot} \mathrm{yr}^{-1}\right)^{1 / 2}$ and $\dot{M}_{w} \approx 2.4 f_{c} N_{22}\left(r_{w} / 10^{16} \mathrm{~cm}\right)^{1 / 2}\left(\dot{M}_{\bullet} / M_{\odot} \mathrm{yr}^{-1}\right)^{1 / 2} M_{\odot} \mathrm{yr}^{-1}$, respectively; here $c$ is the speed of light, $f_{c}$ is the covering factor, $N_{22}$ is the hydrogen column density normalized to $10^{22} \mathrm{~cm}^{-2}$, and $r_{w}$ is the launching radius. Then the kinetic luminosity of the outflow reads

$$
L_{K}=\frac{1}{2} \dot{M}_{w} v_{w}^{2} \approx 2.5 \times 10^{44}\left(\frac{f_{c}}{0.1}\right)\left(\frac{N_{22}}{10}\right)\left(\frac{r_{w}}{10^{16} \mathrm{~cm}}\right)^{-1 / 2}\left(\frac{\dot{M}_{\bullet}}{M_{\odot} / \mathrm{yr}}\right)^{3 / 2} \mathrm{erg} \mathrm{s}^{-1} .
$$

Eventually, QSO outflows remove gas from both the hot and the cold phases at the rates

$$
\dot{M}_{\text {inf,cold }}^{Q S O}=2 \times 10^{3} \epsilon_{Q S O}\left(\frac{V_{\text {vir }}}{500 \mathrm{~km} / \mathrm{s}}\right)^{-2}\left(\frac{\dot{M_{\bullet}}}{M_{\odot} / \mathrm{yr}}\right)^{3 / 2} \frac{M_{\text {inf,cold }}}{M_{\text {cold }}+M_{\text {inf }}} M_{\odot} \mathrm{yr}^{-1},
$$

with $\epsilon_{Q S O}=\left(f_{h} / 0.5\right)\left(f_{c} / 0.1\right)\left(N_{22} / 10\right)$, where $f_{h}$ is the fraction of the kinetic luminosity that couples to the gas. Note that most of the power $L_{h}=f_{h} L_{K}$ is transferred to the gas near the peak of the BH accretion rate curve. Such a power is only a small fraction of the Eddington luminosity $\left(L_{h} / L_{\text {Edd }}\right) \approx 1.4 \times 10^{-2} \epsilon_{Q S O}\left(M_{\bullet} / 10^{8} M_{\odot}\right)$.

The basic outputs of the model are the evolution of the SFR $\dot{M}_{\star}(t)$ and the BH accretion rate $\dot{M}_{\bullet}(t)$ as a function of the galactic age $t$ for any given value of the halo mass $M_{\mathrm{vir}}$ and of the virialization redshift $z_{\mathrm{vir}}$. Once the star formation history of a galaxy has been computed, its luminosity in any chosen band is obtained as a function of $t$ from the GRASIL code, which yields the chemical and the spectro-photometric evolution from the radio to the X-ray band, allowing for the effect of dust absorption and reradiation. 